\title{
QUANTUM STOCHASTIC LIE-TROTTER PRODUCT FORMULA II
}

\author{
J. MARTIN LINDSAY
}

\begin{abstract}
A natural counterpart to the Lie-Trotter product formula for norm-continuous one-parameter semigroups is proved, for the class of quasicontractive quantum stochastic operator cocycles whose expectation semigroup is norm continuous. Compared to previous such results, the assumption of a strong form of independence of the constituent cocycles is overcome. The analysis is facilitated by the development of some quantum Itô algebra. It is also shown how the maximal Gaussian component of a quantum stochastic generator may be extracted — leading to a canonical decomposition of such generators, and the connection to perturbation theory is described. Finally, the quantum Itô algebra is extended to quadratic form generators, and a conjecture is formulated for the extension of the product formula to holomorphic quantum stochastic cocycles.
\end{abstract}

\section{Contents}

\begin{tabular}{|lr}
\hline Introduction & 1 \\
\hline 1. & Quantum Itô algebra \\
\hline 2. & Quantum stochastics \\
\hline 3. & Product Formula \\
\hline 4. & Maximal Gaussian component of a QS generator \\
\hline $5 . \quad$ Perturbation of QS cocycles & 11 \\
\hline $6 . \quad$ Holomorphic QS Cocycles & 15 \\
\hline References & 18 \\
\hline
\end{tabular}

\section{INTRODUCTION}

The Lie product formula in a unital Banach algebra states that

$$
\left(e^{a / n} e^{b / n}\right)^{n} \rightarrow e^{a+b} \text { as } n \rightarrow \infty .
$$

Trotter extended this to $C_{0}$-semigroups on a Banach space where it holds under compatibility assumptions on the generators, convergence being in the strong operator sense ([Tro, see e.g. $[\overline{\mathrm{Dav}}]$ ). It has been further refined, notably by Chernoff ([Che] $)$ and Kato ([Kat] $)$. These product formulae are widely used in mathematical physics and probability theory - for example in establishing positivity preservation of semigroups, and they have an intimate connection to Feynman-Kac formulae (see e.g. [ReS]). Given that quantum stochastic cocycles may be analysed from their associated semigroups ([$\left[\mathrm{LW}_{3}\right)$, it is natural to seek product formulae in this context. Further motivation comes from the fact that

2000 Mathematics Subject Classification. 46L53 (primary); 81S25, 47D06, 46N50 (secondary).

Key words and phrases. Lie-Trotter product formula, quantum stochastic cocycle, one-parameter semigroup, series product, concatenation product, quantum Itô algebra, quantum stochastic analysis. 
such cocycles are quantum counterparts to stochastic semigroups in the sense of Skorohod ([Sko $)$. Product formulae have been obtained in a variety of quantum stochastic settings ([PaS $,\left[\mathrm{LS}_{1,3}\right],[\mathrm{DLT}$, , DGS $)$. The earliest of these dates from before the advent of quantum stochastic calculus $([\mathrm{HuP}])$. In all of these works the constituent cocycles enjoy a strong independence property, namely their respective noise dimension spaces are mutually orthogonal.

In this paper a Lie-Trotter product formula is established for quasicontractive elementary (i.e. Markov-regular) quantum stochastic operator cocycles, with no independence assumption on the driving quantum noise. It is a direct generalisation of the product formula proved in $\left[\mathrm{LS}_{1}\right.$, and is proved by quite different means. Properties of the composition law on the set of quantum stochastic generators that is realised by the stochastic product formula established here are also elucidated. Known in the setting of quantum control theory as the series product $([\mathrm{GoJ}])$, it is more commonly associated with the perturbation of quantum stochastic cocycles $([\mathrm{EvH}])$. The composition of stochastic generators also corresponds to the operator product (i.e. standard composition) of the generators of the quantum random walks whose scaled embeddings approximate the constituent cocycles ([BGL] $)$. Analysis of this composition leads to left and right series decompositions of a quantum stochastic generator. A decomposition for such generators in terms of the socalled concatenation product is also given; this yields the generator's maximal Gaussian part.

It is conjectured here that, as in the case of orthogonal noises $\left(\left[\mathrm{LS}_{3}\right)\right.$, the more general Lie-Trotter product formula given in this paper has an extension to the class of holomorphic quantum stochastic cocycles $\left(\left[\mathrm{LS}_{2}\right]\right)$. By contrast, without orthogonality of noise dimension spaces there seems to be no sensible formulation of a Lie-Trotter product formula for quantum stochastic mapping cocycles.

The plan of the paper is as follows. In Section 1 some quantum Itô algebra is developed, for studying the series product on the class of stochastic generators considered here. In Section 2 the relevant quantum stochastic analysis is recalled. The quantum stochastic Lie-Trotter product formula is proved in Section 3 . In Section 4 the maximal Gaussian component of a quantum stochastic generator is extracted by means of the concatenation product. In the short Section 5, the connection to perturbation theory is described, and in Section 6 the quantum Itô algebra is extended to quadratic form generators and a conjecture for quasicontractive holomorphic quantum stochastic cocycles is formulated.

Notation. For a vector-valued function $f: \mathbb{R}_{+} \rightarrow V$ and subinterval $J$ of $\mathbb{R}_{+}, f_{J}$ denotes the function $\mathbb{R}_{+} \rightarrow V$ which agrees with $f$ on $J$ and vanishes elsewhere. For Hilbert spaces $\mathrm{h}$ and $\mathrm{h}^{\prime}, B\left(\mathrm{~h} ; \mathrm{h}^{\prime}\right)$ denotes the space of bounded operators from $\mathrm{h}$ to $\mathrm{h}^{\prime}$ and $B\left(\mathrm{~h} ; \mathrm{h}^{\prime}\right)_{1}$ denotes its closed unit ball, abbreviated to $B(\mathrm{~h})$ and $B(\mathrm{~h})_{1}$ respectively when $\mathrm{h}^{\prime}=\mathrm{h}$. For an operator $T \in B(\mathrm{~h})$, its real and imaginary parts are denoted $\operatorname{Re} T$ and $\operatorname{Im} T$ respectively, thus $T$ is dissipative if and only if $\operatorname{Re} T \leqslant 0$. The selfadjoint part of a subset $A$ of an involutive space is denoted $A_{\text {sa }}$. The predual of $B(\mathrm{~h})$, that is the space of ultraweakly continuous linear functionals on $B(\mathrm{~h})$, is denoted $B(\mathrm{~h})_{*}$. Algebraic and ultraweak tensor products are denoted by $\underline{\otimes}$ and $\bar{\otimes}$ respectively and, for vectors $\zeta, \eta \in \mathrm{h}$, $\omega_{\zeta, \eta} \in B(\mathrm{~h})_{*}$ denotes the functional given by $T \mapsto\langle\zeta, T \eta\rangle$. The symbol $\subset \subset$ is used to denote finite subset.

\section{Quantum Itô ALGebra}

For this section take Hilbert spaces $\mathrm{h}$ and $\mathrm{H}$. The block matrix decomposition enjoyed by operators in $B(\mathrm{~h} \oplus \mathrm{H})$ is frequently appealed to below. With respect to the distinguished orthogonal projection

$$
\Delta:=P_{\left\{0_{\mathrm{h}}\right\} \oplus \mathrm{H}}=\left[\begin{array}{cc}
0_{\mathrm{h}} & 0_{\mathrm{H} ; \mathrm{h}} \\
0_{\mathrm{h} ; \mathrm{H}} & I_{\mathrm{H}}
\end{array}\right] \in B(\mathrm{~h} \oplus \mathrm{H}),
$$


the composition law on $B(\mathrm{~h} \oplus \mathrm{H})$ given by

$$
F_{1} \triangleleft F_{2}:=F_{1}+F_{1} \Delta F_{2}+F_{2},
$$

with useful alternative asymmetric expressions

$$
F_{1}\left(I+\Delta F_{2}\right)+F_{2} \text { and } F_{1}+\left(I+F_{1} \Delta\right) F_{2},
$$

has both

$$
F_{1}+F_{2}+F_{3}+\left(F_{1} \Delta F_{2}+F_{1} \Delta F_{3}+F_{2} \Delta F_{3}\right)+F_{1} \Delta F_{2} \Delta F_{3},
$$

and

$$
F_{1} \triangleleft F_{3}+\left(I+F_{1} \Delta\right) F_{2}\left(\Delta F_{3}+I\right),
$$

as common expression for $F_{1} \triangleleft\left(F_{2} \triangleleft F_{3}\right)$ and $\left(F_{1} \triangleleft F_{2}\right) \triangleleft F_{3}$. Moreover the composition $\triangleleft$ has $0_{\mathrm{h} \oplus \mathrm{H}}$ as identity element, and the operator adjoint as involution since

$$
\left(F_{1} \triangleleft F_{2}\right)^{*}=F_{2}^{*} \triangleleft F_{1}^{*} .
$$

The notation $\triangleleft$ is taken from the quantum control theory literature, where the composition is called the series product.

Let $\mathfrak{b}(\mathrm{h} \oplus \mathbf{H}):=(B(\mathrm{~h} \oplus \mathbf{H}), \triangleleft)$ denote the resulting *-monoid (i.e. involutive semigroupwith-identity), let $\beta \in \mathbb{R}$, and consider the following subsets of $\mathfrak{b}(\mathrm{h} \oplus \mathrm{H})$ :

$$
\begin{aligned}
\mathfrak{z}(\mathrm{h} \oplus \mathrm{H}) & :=\left\{K \oplus 0_{\mathrm{H}}: K \in B(\mathrm{~h})\right\}=\Delta^{\perp} \mathfrak{b}(\mathrm{h} \oplus \mathrm{H}) \Delta^{\perp}, \\
\mathfrak{c}(\mathrm{h} \oplus \mathrm{H}) & :=\left\{F \in \mathfrak{b}(\mathrm{h} \oplus \mathrm{H}): F^{*} \triangleleft F \leqslant 0\right\}, \\
\mathfrak{q} \mathfrak{c}_{\beta}(\mathrm{h} \oplus \mathrm{H}) & :=\mathfrak{c}(\mathrm{h} \oplus \mathrm{H})+\beta \Delta^{\perp}, \\
\mathfrak{q} \mathfrak{c}(\mathrm{h} \oplus \mathrm{H}) & :=\mathfrak{c}(\mathrm{h} \oplus \mathrm{H})+\mathbb{R}_{+} \Delta^{\perp}, \\
\mathfrak{i}(\mathrm{h} \oplus \mathrm{H}) & :=\left\{F \in \mathfrak{b}(\mathrm{h} \oplus \mathrm{H}): F^{*} \triangleleft F=0\right\}, \\
\mathfrak{i}(\mathrm{h} \oplus \mathrm{H})^{*} & :=\left\{F^{*}: F \in \mathfrak{i}(\mathrm{h} \oplus \mathrm{H})\right\}, \\
\mathfrak{u}(\mathrm{h} \oplus \mathrm{H}) & :=\mathfrak{i}(\mathrm{h} \oplus \mathrm{H}) \cap \mathfrak{i}(\mathrm{h} \oplus \mathrm{H})^{*},
\end{aligned}
$$

and for $F \in \mathfrak{q} \mathfrak{c}(\mathrm{h} \oplus \mathrm{H})$ set

$$
\beta_{0}(F):=\inf \left\{\beta \in \mathbb{R}: F-\beta \Delta^{\perp} \in \mathfrak{c}(\mathbf{h} \oplus \mathrm{H})\right\} .
$$

These classes are relevant to the characterisation of the stochastic generators of quantum stochastic cocycles which are respectively contractive, quasicontractive (with exponential growth bound $\beta_{0}(F)$ ), isometric, coisometric and unitary (see Theorem 2.1 below). Note that, for $F \in \mathfrak{q c}(\mathrm{h} \oplus \mathrm{H})$,

$$
F \in \mathfrak{q c} \mathfrak{c}_{\beta}(\mathrm{h} \oplus \mathrm{H}) \text { if and only if } \beta \geqslant \beta_{0}(F) .
$$

Remarks. Further classes are relevant to the characterisation of quantum stochastic cocycles which are nonnegative, selfadjoint, partially isometric or projection-valued ([Wil]). The characterisation of the generators of 'pure-noise' (or 'local') nonnegative contraction cocycles (for which $h=\mathbb{C}$ ) plays an important role in the identification of the minimal dilation of a quantum dynamical semigroup ([Bha]).

Let $F_{1}, F_{2}$ and $F$ in $\mathfrak{b}(\mathrm{h} \oplus \mathrm{H})$ have respective block matrix forms $\left[\begin{array}{cc}K_{1} & M_{1} \\ L_{1} & Q_{1}-I\end{array}\right],\left[\begin{array}{cc}K_{2} & M_{2} \\ L_{2} & Q_{2}-I\end{array}\right]$ and $\left[\begin{array}{cc}K & M \\ L & Q-I\end{array}\right]$. Then

$$
\begin{aligned}
& F_{1} \triangleleft F_{2}=\left[\begin{array}{cc}
K_{1}+K_{2}+M_{1} L_{2} & M_{1} Q_{2}+M_{2} \\
L_{1}+Q_{1} L_{2} & Q_{1} Q_{2}-I
\end{array}\right], \text { and } \\
& F^{*} \triangleleft F=\left[\begin{array}{cc}
K^{*}+K+L^{*} L & L^{*} Q+M \\
M^{*}+Q^{*} L & Q^{*} Q-I
\end{array}\right],
\end{aligned}
$$


moreover, for $Z \in \mathfrak{z}(\mathrm{h} \oplus \mathrm{H}), \beta \in \mathbb{R}$ and $X \in \mathfrak{b}(\mathrm{h} \oplus \mathrm{H})$,

$$
\begin{aligned}
(F+Z)^{*} \triangleleft(F+Z) & =Z^{*}+F^{*} \triangleleft F+Z \\
\left(F-\beta \Delta^{\perp}\right)^{*} \triangleleft\left(F-\beta \Delta^{\perp}\right) & =F^{*} \triangleleft F-2 \beta \Delta^{\perp}, \text { and } \\
F^{*} \triangleleft X \triangleleft F & =F^{*} \triangleleft F+(I+\Delta F)^{*} X(I+\Delta F) .
\end{aligned}
$$

These identities imply the following relations:

$$
(G \triangleleft F)^{*} \triangleleft(G \triangleleft F) \begin{cases}=F^{*} \triangleleft F+G^{*}+G & \text { if } G \in \mathfrak{z}(\mathbf{h} \oplus \mathbf{H}), \\ \leqslant F^{*} \triangleleft F+2 \beta \Delta^{\perp} & \text { if } G \in \mathfrak{q} \mathfrak{c}_{\beta}(\mathbf{h} \oplus \mathbf{H}), \\ =F^{*} \triangleleft F & \text { if } G \in \mathfrak{i}(\mathrm{h} \oplus \mathrm{H}) .\end{cases}
$$

The basic algebraic properties of these subsets of $\mathfrak{b}(\mathrm{h} \oplus \mathrm{H})$ are collected in the following proposition; using the above observations, their proof is straightforward. Recall that an operator $T \in B(\mathrm{~h})$ is dissipative if it satisfies $\operatorname{Re} T \leqslant 0$, that is

$$
\operatorname{Re}\langle u, T u\rangle \leqslant 0 \quad(u \in \mathrm{h}) .
$$

Proposition 1.1. In the ${ }^{*}$-monoid $\mathfrak{b}(\mathrm{h} \oplus \mathrm{H})$ the following hold.

(a) Its group of invertible elements is given by

$$
\mathfrak{b}(\mathrm{h} \oplus \mathrm{H})^{\times}=\left\{\left[\begin{array}{ll}
* & * \\
* & Q
\end{array}\right]-\Delta \in \mathfrak{b}(\mathrm{h} \oplus \mathrm{H}): Q \in B(\mathrm{H})^{\times}\right\} ;
$$

the identity element being $0=\left[\begin{array}{ll}0 & 0 \\ 0 & I\end{array}\right]-\Delta$, and the inverse of $\left[\begin{array}{cc}K & M \\ L & Q\end{array}\right]-\Delta$ being

$$
\left[\begin{array}{cc}
M Q^{-1} L-K & -M Q^{-1} \\
-Q^{-1} L & Q^{-1}
\end{array}\right]-\Delta=\left[\begin{array}{c}
-M \\
I
\end{array}\right] Q^{-1}\left[\begin{array}{ll}
-L & I
\end{array}\right]-\left[\begin{array}{cc}
K & 0 \\
0 & I
\end{array}\right]
$$

(b) Its centre is $\mathfrak{z}(\mathrm{h} \oplus \mathrm{H})$.

(c) Denoting the class of dissipative operators on $\mathrm{h}$ by $D(\mathrm{~h})$,

$$
\begin{aligned}
& \mathfrak{q} \mathfrak{c}(\mathbf{h} \oplus \mathrm{H})=\mathfrak{c}(\mathbf{h} \oplus \mathrm{H})+\mathfrak{z}(\mathbf{h} \oplus \mathrm{H}), \text { and } \\
& \mathfrak{c}(\mathrm{h} \oplus \mathrm{H}) \cap \mathfrak{z}(\mathrm{h} \oplus \mathrm{H})=\left\{T \oplus 0_{\mathrm{H}}: T \in D(\mathrm{~h})\right\} .
\end{aligned}
$$

(d) $\mathfrak{q} \mathfrak{c}(\mathrm{h} \oplus \mathrm{H}), \mathfrak{c}(\mathrm{h} \oplus \mathrm{H}), \mathfrak{i}(\mathrm{h} \oplus \mathrm{H})$ and $\mathfrak{u}(\mathrm{h} \oplus \mathrm{H})$ are submonoids; their groups of invertible elements are given by

$$
\begin{aligned}
& \mathfrak{i}(\mathrm{h} \oplus \mathrm{H})^{\times}=\mathfrak{c}(\mathrm{h} \oplus \mathrm{H})^{\times}=\mathfrak{u}(\mathrm{h} \oplus \mathrm{H}), \text { and } \\
& \mathfrak{q} \mathfrak{c}(\mathrm{h} \oplus \mathrm{H})^{\times}=\mathfrak{u}(\mathrm{h} \oplus \mathrm{H})+\mathfrak{z}(\mathrm{h} \oplus \mathrm{H}) .
\end{aligned}
$$

Moreover,

$$
\mathfrak{i}(\mathrm{h} \oplus \mathrm{H}) \cap \mathfrak{z}(\mathrm{h} \oplus \mathrm{H})=\mathfrak{u}(\mathrm{h} \oplus \mathrm{H}) \cap \mathfrak{z}(\mathrm{h} \oplus \mathrm{H})=i_{\mathfrak{z}}(\mathrm{h} \oplus \mathrm{H})_{\mathrm{sa}} .
$$

Remark. Clearly $\mathfrak{u}(\mathrm{h} \oplus \mathrm{H})$ is also closed under taking adjoints, and so is a sub-*-monoid of $\mathfrak{b}(\mathrm{h} \oplus \mathrm{H})$.

In fact $\mathfrak{q} \mathfrak{c}(\mathbf{h} \oplus \mathrm{H})$ and $\mathfrak{c}(\mathbf{h} \oplus \mathrm{H})$ are sub-*-monoids too; this is not immediately obvious. It follows from Part (e) of Theorem 1.3 below, but there are now also direct proofs. The one given below is based on an elegant argument of Wills, arising as a biproduct of his analysis of partially isometric quantum stochastic cocycles ([Wil]).

Proposition 1.2. Let $F \in \mathfrak{b}(\mathrm{h} \oplus \mathrm{H})$ and $T \in \mathfrak{z}(\mathrm{h} \oplus \mathrm{H})_{\text {sa }}$. Then

$$
(\Delta F+I)^{*}\left(F \triangleleft F^{*}\right)(\Delta F+I)=F^{*} \triangleleft F+\left(F^{*} \triangleleft F\right) \Delta\left(F^{*} \triangleleft F\right),
$$

and

$$
F^{*} \triangleleft F \leqslant T \text { if and only if } F \triangleleft F^{*} \leqslant T \text {. }
$$


Proof. By the associativity of $\triangleleft$, setting $F_{1}=F^{*}, F_{2}=F \triangleleft F^{*}$ and $F_{3}=F$ in the expression $1.1 \mathrm{~b}$ for $F_{1} \triangleleft F_{2} \triangleleft F_{3}$ we see that

$$
\begin{aligned}
\left.F^{*} \triangleleft F+\operatorname{LHS} 1.3\right) & =F^{*} \triangleleft\left(F \triangleleft F^{*}\right) \triangleleft F \\
& \left.=\left(F^{*} \triangleleft F\right) \triangleleft\left(F^{*} \triangleleft F\right)=\operatorname{RHS} 1.3\right)+F^{*} \triangleleft F,
\end{aligned}
$$

so identity $(1.3)$ holds. Suppose now that $F \triangleleft F^{*} \leqslant T$. Then, since $\Delta T=0=T \Delta$, LHS 1.3$) \leqslant T$ and so, since $\Delta \geqslant 0$ and $F^{*} \triangleleft F$ is selfadjoint,

$$
\left.F^{*} \triangleleft F=1.3\right)-\left(F^{*} \triangleleft F\right) \Delta\left(F^{*} \triangleleft F\right) \leqslant(1.3) \leqslant T .
$$

The converse implication follows by exchanging $F$ and $F^{*}$.

Consider the following possible block matrix forms for $F \in \mathfrak{b}(\mathrm{h} \oplus \mathrm{H})$ :

$$
\begin{aligned}
& {\left[\begin{array}{cc}
\beta I+i H-\frac{1}{2}\left(L^{*} L+A^{2}\right) & -L^{*} C-A D\left(I-C^{*} C\right)^{1 / 2} \\
L & C-I
\end{array}\right], \text { and }} \\
& {\left[\begin{array}{cc}
\beta I+i H-\frac{1}{2}\left(M M^{*}+B^{2}\right) & M \\
-C M^{*}-\left(I-C C^{*}\right)^{1 / 2} E^{*} B & C-I
\end{array}\right],}
\end{aligned}
$$

in which $\beta \in \mathbb{R}, H \in B(\mathrm{~h})_{\mathrm{sa}}, A, B \in B(\mathrm{~h})_{+}, C \in B(\mathrm{H})_{1}$ and $D, E \in B(\mathrm{H} ; \mathrm{h})_{1}$, so that $H=\operatorname{Im} K$.

Theorem 1.3 (Cf. $\left[\mathrm{LW}_{1}, \mathrm{GL}+\right)$. Let $\beta \in \mathbb{R}$ and $F \in \mathfrak{b}(\mathrm{h} \oplus \mathrm{H})$.

(a) The following are equivalent:

(i) $F \in \mathfrak{q c}_{\beta}(\mathrm{h} \oplus \mathrm{H})$.

(ii) $F$ has block matrix form 1.4a).

(iii) $F$ has block matrix form (1.4b).

(iv) $F^{*} \in \mathfrak{q c}_{\beta}(\mathrm{h} \oplus \mathrm{H})$.

(b) $F \in \mathfrak{i}(\mathrm{h} \oplus \mathrm{H})$ if and only if $F$ has block matrix form (1.4a), with $\beta=0, A=0$ and $C$ isometric.

(c) $F \in \mathfrak{i}(\mathrm{h} \oplus \mathrm{H})^{*}$ if and only if $F$ has block matrix form $\left.1.4 \mathrm{~b}\right)$, with $\beta=0, B=0$ and $C$ coisometric.

(d) Let $\mathrm{H}_{1} \oplus \mathrm{H}_{2}$ be an orthogonal decomposition of $\mathrm{H}$. Then, with respect to the inclusion $J_{1}: \mathrm{h} \oplus \mathrm{H}_{1} \rightarrow \mathrm{h} \oplus \mathrm{H}$,

$$
J_{1}^{*} \mathfrak{q} \mathfrak{c}_{\beta}(\mathrm{h} \oplus \mathrm{H}) J_{1}=\mathfrak{q} \mathfrak{c}_{\beta}\left(\mathrm{h} \oplus \mathrm{H}_{1}\right) .
$$

(e) Set $\mathrm{H}^{\prime}:=\mathrm{H} \oplus(\mathrm{H} \oplus \mathrm{h})$. Then, in terms of the inclusion $J: \mathrm{h} \oplus \mathrm{H} \rightarrow(\mathrm{h} \oplus \mathrm{H}) \oplus(\mathrm{H} \oplus \mathrm{h})=$ $\mathrm{h} \oplus \mathrm{H}^{\prime}$,

$$
\mathfrak{c}(\mathrm{h} \oplus \mathrm{H})=J^{*} \mathfrak{u}\left(\mathrm{h} \oplus \mathrm{H}^{\prime}\right) J .
$$

Remark. In block matrix form

$$
J_{1}=I_{\mathrm{h}} \oplus\left[\begin{array}{c}
I_{\mathrm{H}_{1}} \\
0_{\mathrm{H}_{1} ; \mathrm{H}_{2}}
\end{array}\right] \in B\left(\mathbf{h} \oplus \mathrm{H}_{1} ; \mathbf{h} \oplus \mathbf{H}\right), \quad \text { and } \quad J=I_{\mathbf{h}} \oplus\left[\begin{array}{c}
I_{\mathrm{H}} \\
0_{\mathrm{H} ; \mathrm{H} \oplus \mathrm{h}}
\end{array}\right] \in B\left(\mathbf{h} \oplus \mathbf{H} ; \mathbf{h} \oplus \mathbf{H}^{\prime}\right) \text {. }
$$

Proof. The proof exploits the fact that, for an operator $T \in B(\mathrm{~h} \oplus \mathrm{H}), T \geqslant 0$ if and only if $T$ has block matrix form $\left[\begin{array}{c}X \\ Z^{1 / 2} V^{*} X^{1 / 2} \\ X^{1 / 2} V Z^{1 / 2}\end{array}\right]$ where $(X, Z, V) \in B(\mathrm{~h})_{+} \times B(\mathrm{H})_{+} \times$ $B(\mathrm{H} ; \mathrm{h})_{1}$.

(a) Set $G=F-\beta \Delta^{\perp}$. Suppose first that (ii) holds. Then $G$ is given by $1.4 \mathrm{a}$ with $\beta=0$ and, setting $S:=\left(I-C^{*} C\right)^{1 / 2}$,

$$
-G^{*} \triangleleft G=\left[\begin{array}{cc}
A^{2} & A D S \\
S D^{*} A & S^{2}
\end{array}\right] \geqslant 0,
$$


so $G \in \mathfrak{c}(\mathrm{h} \oplus \mathrm{H})$. Thus (ii) implies (i). Conversely, suppose that (i) holds and let $\left[\begin{array}{cc}K & M \\ L & C-I\end{array}\right]$ be the block matrix decomposition of $G$. Then $G \in \mathfrak{c}(\mathrm{h} \oplus \mathrm{H})$ so

$$
\left[\begin{array}{cc}
K^{*}+K+L^{*} L & M+L^{*} C \\
M^{*}+C^{*} L & C^{*} C-I
\end{array}\right]=G^{*} \triangleleft G \leqslant 0 .
$$

Thus $\left(K^{*}+K+L^{*} L\right) \leqslant 0,\|C\| \leqslant 1$ and, for some contraction operator $D$,

$$
M=-L^{*} C-\left[-\left(K^{*}+K+L^{*} L\right)\right]^{1 / 2} D\left(I-C^{*} C\right)^{1 / 2}
$$

so $G$ is given by $1.4 \mathrm{a}$ ) with $\beta=0$ and $H=\operatorname{Im} K$. Thus (i) implies (ii).

Therefore (i) and (ii) are equivalent; taking adjoints we see that (iv) and (iii) are equivalent too. The equivalence of (i) and (iv) follows from Proposition 1.2 .

(b) This is an immediate consequence of (1.2).

(c) This follows from Part (b), by taking adjoints.

(d) For $F_{1} \in \mathfrak{q c}_{\beta}\left(\mathrm{h} \oplus \mathrm{H}_{1}\right)$,

$$
F_{1}=J_{1}^{*} F J_{1}, \quad \text { where } F=\left[\begin{array}{cc}
F_{1} & 0 \\
0 & 0
\end{array}\right] \in \mathfrak{q c}_{\beta}(\mathrm{h} \oplus \mathrm{H}),
$$

so RHS $\subset$ LHS in 1.5). For the reverse inclusion, setting $\Delta_{1}:=0_{\mathrm{h}} \oplus I_{\mathrm{H}_{1}}$ and $\Delta:=0_{\mathrm{h}} \oplus I_{\mathrm{H}}$,

$$
\Delta-J_{1} \Delta_{1} J_{1}^{*}=0_{\mathrm{h}} \oplus I_{\mathrm{H}_{1}} \oplus I_{\mathrm{H}_{2}}-0_{\mathrm{h}} \oplus I_{\mathrm{H}_{1}} \oplus 0_{\mathrm{H}_{2}}=0_{\mathrm{h}} \oplus 0_{\mathrm{H}_{1}} \oplus I_{\mathrm{H}_{2}} \geqslant 0 .
$$

Thus, for $F \in \mathfrak{c}(\mathrm{h} \oplus \mathrm{H})$, the operator $F_{1}:=J_{1}^{*} F J_{1}$ is in $\mathfrak{c}\left(\mathrm{h} \oplus \mathrm{H}_{1}\right)$ since

$$
F_{1}^{*} \triangleleft F_{1}=J_{1}^{*} F^{*} J_{1}+J_{1}^{*} F J_{1}+J_{1}^{*} F^{*} J_{1} \Delta_{1} J_{1}^{*} F J_{1} \leqslant J_{1}^{*}\left(F^{*} \triangleleft F\right) J_{1} \leqslant 0 .
$$

Therefore $J_{1}^{*} \mathfrak{c}(\mathrm{h} \oplus \mathrm{H}) J_{1} \subset \mathfrak{c}\left(\mathrm{h} \oplus \mathrm{H}_{1}\right)$ and so, since $J_{1}^{*} \beta \Delta^{\perp} J_{1}=\beta \Delta_{1}^{\perp}(\beta \in \mathbb{R})$, LHS $\subset$ RHS.

(e) In view of (d), it suffices to show that $J^{*} \mathfrak{u}\left(\mathrm{h} \oplus \mathrm{H}^{\prime}\right) J \supset \mathfrak{c}(\mathbf{h} \oplus \mathbf{H})$. Accordingly, let $F \in \mathfrak{c}(\mathrm{h} \oplus \mathrm{H})$. Then, by what we have proved already, $F$ has block matrix form (1.4a) with $\beta=0$. Setting

$$
F^{\prime}:=\left[\begin{array}{cccc}
i H-\frac{1}{2}\left(L^{*} L+A^{2}\right) & -\left(L^{*} C+A D S\right) & L^{*} T-A D C^{*} & -A R \\
L & C-I & -T & 0 \\
D^{*} A & S & C^{*}-I & 0 \\
R A & 0 & 0 & 0
\end{array}\right],
$$

where $R=\left(I-D D^{*}\right)^{1 / 2}, S=\left(I-C^{*} C\right)^{1 / 2}$, and $T=\left(I-C C^{*}\right)^{1 / 2}$, it is now easily verified that $F^{\prime} \in \mathfrak{u}\left(\mathrm{h} \oplus \mathrm{H}^{\prime}\right)$. Since $J^{*} F^{\prime} J=F$, (e) follows and the proof is complete.

Remarks. The dilation property (e) is effectively proved in [GLt, under the assumption that $\mathrm{h}$ and $\mathrm{H}$ are separable. If $\mathrm{H}=\mathrm{h} \otimes \mathrm{k}$ for a Hilbert space $\mathrm{k}$ (as it is in the application to QS analysis, where $\mathrm{k}$ is the noise dimension space) then, in (e), $\mathrm{H}^{\prime}=\mathrm{h} \otimes \mathrm{k}^{\prime}$ where $\mathrm{k}^{\prime}=\mathrm{k} \oplus \mathrm{k} \oplus \mathbb{C}$.

In terms of its block matrix form $\left[\begin{array}{cc}K & M \\ L & C-I\end{array}\right]$, and bound $\beta_{0}:=\beta_{0}(F)$, the equivalent conditions for $F \in \mathfrak{b}(\mathrm{h} \oplus \mathrm{H})$ to be in $\mathfrak{q} \mathfrak{c}(\mathrm{h} \oplus \mathrm{H})$ read respectively as follows:

$$
\begin{aligned}
& {\left[\begin{array}{cc}
2\left(\operatorname{Re} K-\beta_{0} I_{\mathrm{h}}\right)+L^{*} L & M+L^{*} C \\
M^{*}+C^{*} L & C^{*} C-I_{\mathrm{H}}
\end{array}\right] \leqslant 0, \text { and }} \\
& {\left[\begin{array}{cc}
2\left(\operatorname{Re} K-\beta_{0} I_{\mathrm{h}}\right)+M M^{*} & L^{*}+M C^{*} \\
L+C M^{*} & C C^{*}-I_{\mathrm{H}}
\end{array}\right] \leqslant 0 .}
\end{aligned}
$$

Proposition 1.4 (Left and right series decomposition). Let $F=\left[\begin{array}{cc}K \\ L\end{array}{ }_{C-I}^{M}\right] \in \mathfrak{q} \mathfrak{c}(\mathbf{h} \oplus \mathbf{H})$ and set $\beta_{0}=\beta_{0}(F)$. Then, setting

$$
\begin{aligned}
& F_{1}=\left[\begin{array}{cc}
\beta_{0} I_{\mathrm{h}}+i \operatorname{Im} K & 0 \\
0 & 0
\end{array}\right], F_{2}^{\ell}=\left[\begin{array}{cc}
-\frac{1}{2} L^{*} L & -L^{*} \\
L & 0
\end{array}\right], F_{3}^{r}=\left[\begin{array}{cc}
-\frac{1}{2} M M^{*} & M \\
-M^{*} & 0
\end{array}\right], \\
& F_{3}^{\ell}:=\left[\begin{array}{cc}
\operatorname{Re} K+\frac{1}{2} L^{*} L-\beta_{0} I_{\mathrm{h}} & M+L^{*} C \\
0 & C-I
\end{array}\right], \text { and } F_{2}^{r}:=\left[\begin{array}{cc}
\operatorname{Re} K+\frac{1}{2} M M^{*}-\beta_{0} I_{\mathrm{h}} & 0 \\
L+C M^{*} & C-I
\end{array}\right],
\end{aligned}
$$


the following hold:

$$
F_{1} \in \mathfrak{z}(\mathrm{h} \oplus \mathrm{H}), \quad F_{2}^{\ell}, F_{3}^{r} \in \mathfrak{u}(\mathrm{h} \oplus \mathrm{H}), \quad F_{2}^{r}, F_{3}^{\ell}, \in \mathfrak{c}(\mathrm{h} \oplus \mathrm{H}),
$$

and

$$
F_{1} \triangleleft F_{2}^{\ell} \triangleleft F_{3}^{\ell}=F=F_{1} \triangleleft F_{2}^{r} \triangleleft F_{3}^{r},
$$

Proof. It follows from Theorem 1.3 , and the above block matrix inequalities characterising membership of $\mathfrak{q} \mathfrak{c}(\mathrm{h} \oplus \mathrm{H})$, that

$$
F_{2}^{\ell}, F_{3}^{r} \in \mathfrak{u}(\mathrm{h} \oplus \mathrm{H}) \text { and } F_{3}^{\ell}, F_{2}^{r} \in \mathfrak{c}(\mathrm{h} \oplus \mathrm{H}) .
$$

The two series decompositions follow from the identities

$$
F_{1} \Delta F_{2}^{\ell}=0, F_{2}^{\ell} \Delta F_{3}^{\ell}=\left[\begin{array}{cc}
0 & L^{*}-L^{*} C \\
0 & 0
\end{array}\right], F_{1} \Delta F_{2}^{r}=0 \text { and } F_{2}^{r} \Delta F_{3}^{r}=\left[\begin{array}{cc}
0 & 0 \\
M^{*}-C M^{*} & 0
\end{array}\right] .
$$

The rest is clear.

Remarks. (i) The left and right series decompositions are related via the adjoint operation as follows:

$$
\left(F^{*}\right)_{1}=\left(F_{1}\right)^{*},\left(F^{*}\right)_{2}^{\ell}=\left(F_{3}^{r}\right)^{*} \text { and }\left(F^{*}\right)_{3}^{\ell}=\left(F_{2}^{r}\right)^{*} .
$$

(ii) Let $F=\left[\begin{array}{cc}K & M \\ L & M\end{array}\right] \in \mathfrak{q} \mathfrak{c}(\mathrm{h} \oplus \mathrm{H})$ and set $H:=\operatorname{Im} K$. Then $F \in \mathfrak{i}(\mathbf{h} \oplus \mathbf{H})$ if and only if $F$ has left series decomposition

$$
i\left[\begin{array}{cc}
H & 0 \\
0 & 0
\end{array}\right] \triangleleft\left[\begin{array}{cc}
-\frac{1}{2} L^{*} L & -L^{*} \\
L & 0
\end{array}\right] \triangleleft\left[\begin{array}{cc}
0 & 0 \\
0 & C-I
\end{array}\right],
$$

with $C$ isometric, whereas $F \in \mathfrak{i}(\mathrm{h} \oplus \mathrm{H})^{*}$ if and only if $F$ has right series decomposition

$$
i\left[\begin{array}{cc}
H & 0 \\
0 & 0
\end{array}\right] \triangleleft\left[\begin{array}{cc}
0 & 0 \\
0 & C-I
\end{array}\right] \triangleleft\left[\begin{array}{cc}
-\frac{1}{2} M M^{*} & M \\
-M^{*} & 0
\end{array}\right],
$$

with $C$ coisometric.

Now suppose that $\mathrm{H}$ has an orthogonal decomposition $\mathrm{H}_{1} \oplus \mathrm{H}_{2}$. Define injections

$$
\begin{gathered}
\iota: \mathfrak{b}\left(\mathrm{h} \oplus \mathrm{H}_{1}\right) \rightarrow \mathfrak{b}(\mathrm{h} \oplus \mathrm{H}), \quad F=\left[\begin{array}{cc}
K & M \\
L & N
\end{array}\right] \mapsto F \oplus 0_{\mathrm{H}_{2}}=\left[\begin{array}{ccc}
K & M & 0 \\
L & N & 0 \\
0 & 0 & 0
\end{array}\right], \text { and } \\
\iota^{\prime}: \mathfrak{b}\left(\mathrm{h} \oplus \mathrm{H}_{2}\right) \rightarrow \mathfrak{b}(\mathrm{h} \oplus \mathrm{H}), \quad F=\left[\begin{array}{cc}
K & M \\
L & N
\end{array}\right] \mapsto \Sigma\left(F \oplus 0_{\mathrm{H}_{1}}\right)=\left[\begin{array}{ccc}
K & 0 & M \\
0 & 0 & 0 \\
L & 0 & N
\end{array}\right],
\end{gathered}
$$

$\Sigma$ being the sum-flip map $B\left(\mathrm{~h} \oplus \mathrm{H}_{2} \oplus \mathrm{H}_{1}\right) \rightarrow B(\mathrm{~h} \oplus \mathrm{H})$, and define the composition

$$
\mathfrak{b}\left(\mathrm{h} \oplus \mathrm{H}_{1}\right) \times \mathfrak{b}\left(\mathrm{h} \oplus \mathrm{H}_{2}\right) \rightarrow \mathfrak{b}(\mathrm{h} \oplus \mathrm{H}), \quad\left(F_{1}, F_{2}\right) \mapsto F_{1} \boxplus F_{2}:=\iota\left(F_{1}\right)+\iota^{\prime}\left(F_{2}\right),
$$

known as the concatenation product in quantum control theory ([GoJ]). Thus

$$
\left[\begin{array}{cc}
K_{1} & M_{1} \\
L_{1} & N_{1}
\end{array}\right] \boxplus\left[\begin{array}{cc}
K_{2} & M_{2} \\
L_{2} & N_{2}
\end{array}\right]=\left[\begin{array}{ccc}
K_{1}+K_{2} & M_{1} & M_{2} \\
L_{1} & N_{1} & 0 \\
L_{2} & 0 & N_{2}
\end{array}\right] .
$$

Note that

$$
\iota\left(F_{1}\right)=F_{1} \boxplus 0_{\mathrm{H}_{2}} \text { and } \iota^{\prime}\left(F_{2}\right)=0_{\mathrm{H}_{1}} \boxplus F_{2} .
$$

In view of the identity

$$
\iota\left(F_{1}\right) \Delta \iota^{\prime}\left(F_{2}\right)=0 \mathrm{~h} \oplus \mathbf{H}=\iota^{\prime}\left(F_{2}\right) \Delta \iota\left(F_{1}\right),
$$

for $F_{1} \in \mathfrak{b}\left(\mathrm{h} \oplus \mathrm{H}_{1}\right)$ and $F_{2} \in \mathfrak{b}\left(\mathrm{h} \oplus \mathrm{H}_{2}\right)$, the concatenation product is effectively a special case of the series product:

$$
F_{1} \boxplus F_{2}=\iota\left(F_{1}\right) \triangleleft \iota^{\prime}\left(F_{2}\right) .
$$


We end this section with a significant representation of the quantum Itô algebra. It is relevant to the realisation of QS cocycles as time-ordered exponentials ([Hol] $)$, and also to the convergence of a class of scaled quantum random walks to QS cocycles ([BGL]). Set $\mathcal{S}:=I_{\mathrm{h} \oplus \mathrm{H} \oplus \mathrm{h}}+\mathrm{A}$, where A is the following subalgebra of $B(\mathrm{~h} \oplus \mathrm{H} \oplus \mathrm{h})$ :

$$
\left\{T \in B(\mathrm{~h} \oplus \mathrm{H} \oplus \mathrm{h}): P_{\mathrm{h} \oplus \mathrm{H} \oplus\{0\}} T=T=T P_{\{0\} \oplus \mathrm{H} \oplus \mathrm{h}\}} ;\right.
$$

thus $\mathcal{S}$ consists of the elements of $B(\mathrm{~h} \oplus \mathrm{H} \oplus \mathrm{h})$ having block matrix form $\left[\begin{array}{ccc}I_{\mathrm{h}} & * & * \\ 0 & * & * \\ 0 & 0 & I_{\mathrm{h}}\end{array}\right]$. With respect to (operator composition and) the involution given by

$$
T \mapsto T^{\star}:=\Xi T \Xi \text {, where } \Xi:=\left[\begin{array}{ccc}
0 & 0 & I_{\mathrm{h}} \\
0 & I_{\mathrm{H}} & 0 \\
I_{\mathrm{h}} & 0 & 0
\end{array}\right],
$$

$\mathcal{S}$ is a sub-*-monoid of $B(\mathrm{~h} \oplus \mathrm{H} \oplus \mathrm{h})$, and the following is readily verified.

Proposition 1.5 ([이],$[\mathrm{Bel}])$. The prescription

$$
\left[\begin{array}{cc}
K & M \\
L & Q-I
\end{array}\right] \mapsto\left[\begin{array}{ccc}
I_{\mathrm{h}} & M & K \\
0 & Q & L \\
0 & 0 & I_{\mathrm{h}}
\end{array}\right]=I_{\mathrm{h} \oplus \mathbf{H} \oplus \mathrm{h}}+\left[\begin{array}{ccc}
0 & M & K \\
0 & Q-I & L \\
0 & 0 & 0
\end{array}\right]
$$

defines an isomorphism of ${ }^{*}$-monoids $\phi: \mathfrak{b}(\mathrm{h} \oplus \mathrm{H}) \rightarrow(\mathcal{S}, \cdot, \star)$.

\section{Quantum stochastics}

For the rest of the paper fix Hilbert spaces $\mathfrak{h}$ and $\mathrm{k}$, and set $\widehat{\mathrm{k}}:=\mathbb{C} \oplus \mathrm{k}$ and $\mathrm{K}:=$ $L^{2}\left(\mathbb{R}_{+} ; \mathrm{k}\right)$. The quantum Itô algebra developed in the previous section is applied below with $\mathbf{h}=\mathfrak{h}$ and $\mathbf{H}=\mathbf{k} \otimes \mathfrak{h}$, so that $\mathbf{h} \oplus \mathbf{H}=\widehat{k} \otimes \mathfrak{h}$. In this context, the operator

$$
\Delta=\Delta_{\mathrm{k}}:=P_{\left\{0_{\mathbb{C}}\right\} \oplus \mathrm{k}}=\left[\begin{array}{cc}
0 & 0 \\
0 & I_{\mathrm{k}}
\end{array}\right] \in B(\widehat{\mathrm{k}})
$$

is ubiquitous; below it is freely ampliated.

In this section we collect the quantum stochastic (QS) facts needed below. For more detail, see [L]; for further background, see $[\mathrm{Par}]$ and $[\mathrm{Mey}]$. Let $\mathcal{F}$ denote the symmetric Fock space over K. We use normalised exponential vectors

$$
\varpi(g):=e^{-\|g\|^{2} / 2} \varepsilon(g) \text { where } \varepsilon(g):=\left((n !)^{-1 / 2} g^{\otimes n}\right)_{n \geqslant 0} \quad(g \in \mathrm{K}),
$$

in terms of which the Fock-Weyl operators are the unitary operators on $\mathcal{F}$ determined by the identity

$$
W(f) \varpi(g)=e^{-i \operatorname{Im}\langle f, g\rangle} \varpi(f+g) \quad(f, g \in \mathbf{K}),
$$

and the second quantisation operators are the contractions from $\mathcal{F}$ to $\mathcal{F}^{\prime}$ determined by the identity

$$
\Gamma(C) \varepsilon(g):=\varepsilon(C g) \quad\left(C \in B\left(\mathrm{~K} ; \mathrm{K}^{\prime}\right)_{1}, g \in \mathrm{K}\right),
$$

where $\mathcal{F}^{\prime}$ is the symmetric Fock space over a Hilbert space $\mathrm{K}^{\prime}$. Note that $\Gamma(C)$ is isometric if $C$ is, and $\Gamma\left(C^{*}\right)=\Gamma(C)^{*}$. There is a useful family of slice maps:

$$
\Omega\left(g^{\prime}, g\right):=\mathrm{id}_{B\left(\mathrm{~h} ; \mathrm{h}^{\prime}\right)} \bar{\otimes} \omega_{\varpi\left(g^{\prime}\right), \varpi(g)}: B\left(\mathrm{~h} ; \mathrm{h}^{\prime}\right) \bar{\otimes} B(\mathcal{F}) \rightarrow B\left(\mathrm{~h} ; \mathrm{h}^{\prime}\right) \quad\left(g^{\prime}, g \in \mathrm{K}\right),
$$

amongst which $\mathbb{E}:=\Omega(0,0)$ is referred to as the vacuum expectation. (The Hilbert spaces $\mathrm{h}$ and $\mathrm{h}^{\prime}$ are determined by context.) Moreover, the slice maps extend to unbounded operators from $\mathrm{h} \otimes \mathcal{F}$ to $\mathrm{h}^{\prime} \otimes \mathcal{F}$ whose domains include $\mathrm{h} \underline{\otimes} \mathcal{E}$, where $\mathcal{E}:=\operatorname{Lin}\{\varpi(g): g \in \mathrm{K}\}$. The following obvious identity is exploited below:

$$
\Omega\left(g^{\prime}, g\right)(T)=\mathbb{E}\left[\left(I_{\mathrm{h}^{\prime}} \otimes W\left(g^{\prime}\right)\right)^{*} T\left(I_{\mathrm{h}} \otimes W(g)\right)\right] .
$$

We also need two families of endomorphisms of (the von Neumann algebra) $B(\mathcal{F})$ :

$$
\rho_{t}: T \mapsto R_{t} T R_{t} \text { and } \sigma_{t}: T \mapsto I_{\mathcal{F}_{[0, t]}} \otimes S_{t} T S_{t}^{*} \quad\left(t \in \mathbb{R}_{+}\right),
$$


where $R_{t}:=\Gamma\left(r_{t}\right)$ and $S_{t}:=\Gamma\left(s_{t}\right)$ for the unitary operators $r_{t}$ and $s_{t}$ defined as follows

$$
\begin{aligned}
& r_{t}: \mathrm{K} \rightarrow \mathrm{K}, \quad\left(r_{t} f\right)(s)=\left\{\begin{array}{ll}
f(t-s) & \text { if } s \in[0, t[ \\
f(s) & \text { if } s \in[t, \infty[
\end{array},\right. \\
& s_{t}: \mathrm{K} \rightarrow \mathrm{K}_{[t, \infty[}, \quad\left(s_{t} f\right)(s)=f(s-t),
\end{aligned}
$$

where $\mathrm{K}_{[t, \infty[}:=L^{2}\left(\left[t, \infty[; \mathrm{k})\right.\right.$. The time-reversal maps $\left(\rho_{t}\right)_{t \geqslant 0}$ are obviously involutive: $\rho_{t}^{2}=\mathrm{id}_{B(\mathcal{F})}$, and the time-shift maps $\left(\sigma_{t}\right)_{t \in \mathbb{R}_{+}}$form a semigroup, known as the CCR flow of index $\mathrm{k}$. Both are freely ampliated to act on $B\left(\mathrm{~h} ; \mathrm{h}^{\prime}\right) \bar{\otimes} B(\mathcal{F})$, for Hilbert spaces $\mathrm{h}$ and $h^{\prime}$. For use below, note the identity

$$
\Omega\left(g_{[r, \infty[}^{\prime}, g_{[r, \infty[}\right) \circ \sigma_{r}=\Omega\left(s_{r}^{*}\left(\left.g^{\prime}\right|_{[r, \infty[}\right), s_{r}^{*}\left(\left.g\right|_{[r, \infty[}\right)\right) \quad\left(g, g^{\prime} \in \mathrm{K}, r \in \mathbb{R}_{+}\right) .
$$

Let $X^{i}=\left(X_{0}^{i}+\Lambda_{t}\left(H^{i}\right)\right)_{t \geqslant 0}$ for a QS integrand $\left(\mathrm{h}_{i}, \mathrm{~h}_{i-1}\right)$-process $H^{i}$ and bounded operator $X_{0}^{i} \in B\left(\mathrm{~h}_{i} ; \mathrm{h}_{i-1}\right) \otimes I_{\mathcal{F}}$, where $i=1,2$. Suppose that all of the processes $H^{1}, H^{2}, X^{1}$ and $X^{2}$ are bounded, that is they consist of bounded operators, and $H^{1}$ and $H^{2}$ are strongly continuous. Then the quantum Itô product formula ([HuP $)$ reads

$$
X_{t}^{1} X_{t}^{2}=X_{0}^{1} X_{0}^{2}+\Lambda_{t}(H) \text { where } H=\left(H_{s}^{1}\left(I_{\widehat{\mathrm{k}}} \otimes X_{s}^{2}\right)+\left(I_{\widehat{\mathrm{k}}} \otimes X_{s}^{1}\right) H_{s}^{2}+H_{s}^{1} \Delta H_{s}^{2}\right)_{s \geqslant 0},
$$

with $\Delta$ abbreviating $\Delta \otimes I_{\mathrm{h}_{1} \otimes \mathcal{F}}$.

Let $X=\left(\Lambda_{t}(H)\right)_{t \geqslant 0}$ for a bounded QS integrand $\left(\mathrm{h}_{1}, \mathrm{~h}_{2}\right)$-process $H$ with block matrix form $\left[\begin{array}{cc}K & * \\ * & *\end{array}\right]$, then

$$
\mathbb{E}\left[X_{t}\right] v=\int_{0}^{t} \mathrm{~d} s \mathbb{E}\left[K_{s}\right] v \quad\left(v \in \mathrm{h}_{1}, t \in \mathbb{R}_{+}\right),
$$

and, if the process $X$ is bounded then, for bounded operators $R \in B\left(\mathrm{~h}_{2} ; \mathrm{h}_{3}\right) \otimes I_{\mathcal{F}}$ and $S \in B\left(\mathrm{~h}_{0} ; \mathrm{h}_{1}\right) \otimes I_{\mathcal{F}}$,

$$
R \Lambda_{t}(H) S=\Lambda_{t}\left(\left(I_{\widehat{\mathrm{k}}} \otimes R\right) H .\left(I_{\widehat{\mathrm{k}}} \otimes S\right)\right) \quad\left(t \in \mathbb{R}_{+}\right) .
$$

A bounded $Q S$ (left) cocycle on $\mathfrak{h}$, with noise dimension space $\mathrm{k}$, is a bounded process $V$ on $\mathfrak{h}$ which satisfies

$$
V_{0}=I_{\mathfrak{h} \otimes \mathcal{F}} \text { and } V_{s+t}=V_{s} \sigma_{s}\left(V_{t}\right) \quad\left(s, t \in \mathbb{R}_{+}\right) .
$$

It is a $Q S$ right cocycle if instead it satisfies $V_{s+t}=\sigma_{s}\left(V_{t}\right) V_{s}$ for $s, t \in \mathbb{R}_{+}$. If $V$ is a QS left cocycle then, $V^{*}:=\left(V_{t}^{*}\right)_{t \geqslant 0}$ and $V^{\mathrm{r}}:=\left(\rho_{t}\left(V_{t}\right)\right)_{t \geqslant 0}$ define QS right cocycles, and

$$
V^{\sharp}:=\left(\rho_{t}\left(V_{t}^{*}\right)=\rho\left(V_{t}\right)^{*}\right)_{t \geqslant 0}
$$

defines a QS left cocycle, called the dual cocycle of $V$ ([Jou $]$ ). This said, we work exclusively with QS left cocycles in this paper. Following standard terminology of semigroup theory $([\mathrm{HiP}]$,

$$
\beta_{0}(V):=\inf \left\{\beta \in \mathbb{R}: \sup _{t>0}\left\|e^{-\beta t} V_{t}\right\|<\infty\right\}
$$

is referred to as the exponential growth bound of $V$. If $V$ is strongly continuous then $\beta_{0}(V)<\infty ; V$ is called quasicontractive if, for some $\beta \in \mathbb{R}$, the QS cocycle $\left(e^{-\beta t} V_{t}\right)_{t \geqslant 0}$ is contractive. When $V$ is locally uniformly bounded, it is called elementary (or Markov regular) if its expectation semigroup $\left(\mathbb{E}\left[V_{t}\right]\right)_{t \geqslant 0}$ is norm continuous.

Note that, given a QS cocycle $V$, the two-parameter family $V_{r, t}:=\sigma_{r}\left(V_{t-r}\right)$ satisfies $V_{0, t}=V_{t}\left(t \in \mathbb{R}_{+}\right)$, the evolution equation

$$
V_{r, r}=I_{\mathfrak{h} \otimes \mathcal{F}} \text { and } V_{r, t}=V_{r, s} V_{s, t} \quad(t \geqslant s \geqslant r),
$$

and the biadaptedness property

$$
V_{r, t} \in\left(B(\mathfrak{h}) \otimes I_{[0, r[}\right) \bar{\otimes}\left(B\left(\mathcal{F}_{[r, t]}\right) \otimes I_{[t, \infty[}\right) \quad(t \geqslant r \geqslant 0) .
$$


For use below, note that in the notation $(2.2)$, these $Q S$ evolutions satisfy the timecovariance identity

$$
\Omega\left(c_{[r, t[}^{\prime}, c_{[r, t]}\right)\left(V_{r, t}\right)=\Omega\left(c_{[0, t-r[}^{\prime}, c_{[0, t-r[}\right)\left(V_{t-r}\right)
$$

by (2.3), and further evolution identity

$$
\Omega\left(g_{[r, t]}^{\prime}, g_{[r, t]}\right)\left(V_{r, t}\right)=\Omega\left(g_{[r, s[}^{\prime}, g_{[r, s]}\right)\left(V_{r, s}\right) \Omega\left(g_{[s, t]}^{\prime}, g_{[s, t]}\right)\left(V_{s, t}\right)
$$

for $c^{\prime}, c \in \mathrm{k}, g^{\prime}, g \in L_{\mathrm{loc}}^{2}\left(\mathbb{R}_{+} ; \mathrm{k}\right)$ and $t \geqslant s \geqslant r \geqslant 0$.

Remark. Note that the relations (2.8a) and (2.8b) also hold when $V$ is a product of such QS evolutions.

Theorem 2.1 ([Fag, $\left.\left[\mathrm{LW}_{1,2}\right]\right)$. For $F \in \mathfrak{q} \mathfrak{c}_{\beta}(\widehat{\mathrm{k}} \otimes \mathfrak{h})$ where $\beta \in \mathbb{R}$, the (left) QS differential equation $\mathrm{d} X_{t}=X_{t} \cdot F \mathrm{~d} \Lambda_{t}, X_{0}=I$ has a unique weakly regular weak solution, denoted $X^{F}$, moreover $X^{F}$ is a quasicontractive, elementary $Q S$ cocycle, with exponential growth bound at most $\beta$, strongly satisfying its $Q S$ differential equation. The resulting $\operatorname{map} F \mapsto X^{F}$ is bijective from $\mathfrak{q} \mathfrak{c}(\widehat{\mathrm{k}} \otimes \mathfrak{h})$ to the class of quasicontractive elementary QS cocycles; it restricts to bijections from $\mathfrak{c}(\widehat{\mathrm{k}} \otimes \mathfrak{h}), \mathfrak{i}(\widehat{\mathrm{k}} \otimes \mathfrak{h})$ and $\mathfrak{i}(\widehat{\mathrm{k}} \otimes \mathfrak{h})^{*}$ to the respective subclasses of contractive, isometric and coisometric, elementary $Q S$ cocycles, moreover it satisfies $X^{F^{*}}=\left(X^{F}\right)^{\sharp}$.

The unique operator $F$ associated with a quasicontractive elementary QS cocycle $V$ in this way is referred to as the stochastic generator of $V$.

There is a basic class of QS cocycles which plays an important role.

Example 2.2. For each $c \in \mathrm{k}$, the (Fock) Weyl cocycle $W^{c}$ is given by

$$
W^{c}:=\left(I_{\mathfrak{h}} \otimes W\left(c_{[0, t]}\right)\right)_{t \geqslant 0} \quad(c \in \mathbf{k}) .
$$

These are QS unitary cocycles (both left and right), and $X=W^{c}$ satisfies

$$
X^{\mathrm{r}}=X \text { and } X^{\sharp}=W^{-c}=X^{*},
$$

moreover $X$ is elementary with stochastic generator

$$
F_{c}:=\left[\begin{array}{cc}
-\frac{1}{2}\|c\|^{2} & -\langle c| \\
|c\rangle & 0
\end{array}\right] \otimes I_{\mathfrak{h}} \in B(\widehat{\mathrm{k}} \otimes \mathfrak{h})
$$

Proposition 2.3. Let $F \in \mathfrak{q} \mathfrak{c}(\widehat{\mathrm{k}} \otimes \mathfrak{h})$ and $G \in \mathfrak{q} \mathfrak{c}(\widehat{\mathrm{k}}) \otimes I_{\mathfrak{h}} \subset \mathfrak{q} \mathfrak{c}(\widehat{\mathrm{k}} \otimes \mathfrak{h})$. Then

$$
X^{F} X^{G}=X^{F \triangleleft G} \text { and } X^{G} X^{F}=X^{G \triangleleft F} \text {. }
$$

Proof. First note that, since $X^{G}$ is of the form $\left(I_{\mathfrak{h}} \otimes V_{t}\right)_{t \geqslant 0}$ for a quasicontractive QS cocycle $V$ on $\mathbb{C}$,

$$
\left(F \otimes I_{\mathcal{F}}\right)\left(I_{\widehat{\mathrm{k}}} \otimes X_{s}^{G}\right)=F \otimes V_{s}=\left(I_{\widehat{\mathrm{k}}} \otimes X_{s}^{G}\right)\left(F \otimes I_{\mathcal{F}}\right) \quad\left(s \in \mathbb{R}_{+}\right) .
$$

Therefore, by the quantum Itô product formula (2.4),

$$
\begin{aligned}
\mathrm{d}\left(X_{t}^{F} X_{t}^{G}\right)= & \left(\left(I_{\widehat{\mathrm{k}}} \otimes X_{t}^{F}\right)\left(F \otimes I_{\mathcal{F}}\right)\left(I_{\widehat{\mathrm{k}}} \otimes X_{t}^{G}\right)+\left(I_{\widehat{\mathrm{k}}} \otimes X_{t}^{F} X_{t}^{G}\right)\left(G \otimes I_{\mathcal{F}}\right)\right. \\
& \left.+\left(I_{\widehat{\mathrm{k}}} \otimes X_{t}^{F}\right)\left(F \otimes I_{\mathcal{F}}\right)\left(I_{\widehat{\mathrm{k}}} \otimes X_{t}^{G}\right)\left(\Delta G \otimes I_{\mathcal{F}}\right)\right) \mathrm{d} \Lambda_{t} \\
= & X_{t}^{F} X_{t}^{G} \cdot(F \triangleleft G) \mathrm{d} \Lambda_{t} .
\end{aligned}
$$

Since $X_{0}^{F} X_{0}^{G}=I$ and $F \triangleleft G \in \mathfrak{q} \mathfrak{c}(\widehat{\mathrm{k}} \otimes \mathfrak{h})$, uniqueness for strongly continuous bounded solutions of the QS differential equation

$$
\mathrm{d} X_{t}=X_{t} \cdot(F \triangleleft G) \mathrm{d} \Lambda_{t}, \quad X_{0}=I_{\mathfrak{h} \otimes \mathcal{F}}
$$

(see Theorem 2.1) implies that $X^{F} X^{G}=X^{F \triangleleft G}$. Since $F^{*} \in \mathfrak{q} \mathfrak{c}(\widehat{\mathrm{k}} \otimes \mathfrak{h})$ and $G^{*} \in \mathfrak{q} \mathfrak{c}(\widehat{\mathrm{k}}) \otimes I_{\mathfrak{h}}$, it follows that $X^{F^{*} \triangleleft G^{*}}=X^{F^{*}} X^{G^{*}}$ too. The second identity now follows by duality:

$$
X^{G \triangleleft F}=X^{\left(F^{*} \triangleleft G^{*}\right)^{*}}=\left(X^{F^{*} \triangleleft G^{*}}\right)^{\sharp}=\left(X^{F^{*}} X^{G^{*}}\right)^{\sharp}=\left(X^{G^{*}}\right)^{\sharp}\left(X^{F^{*}}\right)^{\sharp}=X^{G} X^{F} .
$$


Remarks. (i) More generally, if two quasicontractive QS cocycles commute on their initial space then their (pointwise) product is also a cocycle.

(ii) In the light of the identities

$$
F_{c} \triangleleft F_{d}=F_{c+d}-i \operatorname{Im}\langle c, d\rangle \Delta^{\perp} \text { and } F_{-c}=F_{c}^{*} \quad(c, d \in \mathrm{k}),
$$

Proposition 2.3 contains Weyl commutation relations (see $[\mathrm{BrR}]$, or $[\mathrm{L}]$ ) as a special case.

The connection to quantum random walks mentioned in the introduction is as follows (for details, in particular the precise meaning of the terminology used, see [BGL]).

Theorem 2.4 ([BGL] $)$. For $i=1,2$, let $F_{i} \in \mathfrak{q} \mathfrak{c}(\widehat{\mathrm{k}} \otimes \mathfrak{h})$ and let $\left(G_{i}(h)\right)_{h>0}$ be a family in $B(\widehat{\mathrm{k}} \otimes \mathfrak{h})$ satisfying

$$
\begin{aligned}
& \sup \left\{\left\|G_{i}(h)\right\|^{n}: n \in \mathbb{N}, h>0, n h \leqslant T\right\}<\infty \quad\left(T \in \mathbb{R}_{+}\right), \quad \text { and } \\
& \left(h^{-1 / 2} \Delta^{\perp}+\Delta\right)\left(G_{i}(h)-\Delta^{\perp}\right)\left(h^{-1 / 2} \Delta^{\perp}+\Delta\right) \rightarrow F_{i}+\Delta \quad \text { as } h \rightarrow 0 .
\end{aligned}
$$

Then, for all $\varphi \in B(\mathcal{F})_{*}$ and $T \in \mathbb{R}_{+}$,

$$
\sup _{0 \leqslant t \leqslant T}\left\|\left(\operatorname{id}_{B(\mathfrak{h})} \bar{\otimes} \varphi\right)\left(X_{t}^{\langle h\rangle}-X_{t}^{F_{1} \triangleleft F_{2}}\right)\right\| \rightarrow 0 \quad \text { as } h \rightarrow 0,
$$

where, for $h>0,\left(X_{t}^{\langle h\rangle}\right)_{t \geqslant 0}$ denotes the $h$-scale embedded left quantum random walk generated by $G_{1}(h) G_{2}(h)$.

\section{Product Formula}

For proving the quantum stochastic Lie-Trotter product formula it is convenient to define a constant associated with a pair of quantum stochastic generators. Thus, for $F_{1}, F_{2} \in \mathfrak{b}(\widehat{\mathrm{k}} \otimes \mathfrak{h})$ with respective block matrix forms $\left[\begin{array}{ll}K_{1} & M_{1} \\ L_{1} & N_{1}\end{array}\right]$ and $\left[\begin{array}{ll}K_{2} & M_{2} \\ L_{2} & N_{2}\end{array}\right]$, set

$$
\begin{aligned}
C\left(F_{1}, F_{2}\right): & =\left\|\Delta^{\perp} F_{1} \Delta^{\perp}\right\|+\left\|\Delta^{\perp} F_{2} \Delta^{\perp}\right\|+\left\|\Delta^{\perp} F_{1} \Delta\right\| \cdot\left\|\Delta F_{2} \Delta^{\perp}\right\| \\
& =\left\|K_{1}\right\|+\left\|K_{2}\right\|+\left\|M_{1}\right\|\left\|L_{2}\right\| .
\end{aligned}
$$

Note that this depends only on the first row of $F_{1}$ and first column of $F_{2}$ :

$$
C\left(F_{1}, F_{2}\right)=C\left(\Delta^{\perp} F_{1}, F_{2} \Delta^{\perp}\right) .
$$

Proposition 3.1. Let $F_{i} \in \mathfrak{q c}_{\beta_{i}}(\widehat{\mathrm{k}} \otimes \mathfrak{h})$ with block matrix form $\left[\begin{array}{ll}K_{i} & M_{i} \\ L_{i} & N_{i}\end{array}\right]$, for $i=1,2$. Then

$$
\left\|\mathbb{E}\left[X_{t}^{F_{1}} X_{t}^{F_{2}}-X_{t}^{F_{1} \triangleleft F_{2}}\right]\right\| \leqslant t^{2} e^{t\left(\beta_{1}+\beta_{2}\right)} C\left(F_{1}, F_{2}\right)^{2} \quad\left(t \in \mathbb{R}_{+}\right) .
$$

Proof. Set $V^{(1)}=X^{F_{1}}, V^{(2)}=X^{F_{2}}$ and $V=X^{F_{1} \triangleleft F_{2}}$. Since

$$
e^{-t\left(\beta_{1}+\beta_{2}\right)}\left(V_{t}^{(1)} V_{t}^{(2)}-V_{t}\right)=X_{t}^{G_{1}} X_{t}^{G_{2}}-X_{t}^{G_{1} \triangleleft G_{2}} \quad\left(t \in \mathbb{R}_{+}\right),
$$

where $G_{i}=F_{i}-\beta_{i} \Delta^{\perp} \in \mathfrak{c}(\widehat{\mathrm{k}} \otimes \mathfrak{h})(i=1,2)$, it suffices to assume that $\beta_{1}=\beta_{2}=0$, so that $F_{1}, F_{2}, F_{1} \triangleleft F_{2} \in \mathfrak{c}(\widehat{\mathrm{k}} \otimes \mathfrak{h})$, and the QS cocycles $V^{(1)}, V^{(2)}$ and $V$ are all contractive.

Fix $t \in \mathbb{R}_{+}$. By the quantum Itô product formula (2.4), $V^{(1)} V^{(2)}-V=\Lambda$. $(H)$ where $H=H^{(1)}+H^{(2)}+H^{(3)}$ for the processes given by

$$
\begin{aligned}
H_{s}^{(1)} & :=\left(I_{\widehat{\mathrm{k}}} \otimes V_{s}^{(1)}\right)\left(F_{1} \otimes I_{\mathcal{F}}\right)\left(I_{\widehat{\mathrm{k}}} \otimes V_{s}^{(2)}\right)-\left(I_{\widehat{\mathrm{k}}} \otimes V_{s}\right)\left(F_{1} \otimes I_{\mathcal{F}}\right) \\
H_{s}^{(2)} & :=\left(I_{\widehat{\mathrm{k}}} \otimes\left(V_{s}^{(1)} V_{s}^{(2)}-V_{s}\right)\right)\left(F_{2} \otimes I_{\mathcal{F}}\right) \\
H_{s}^{(3)} & :=\left(I_{\widehat{\mathrm{k}}} \otimes V_{s}^{(1)}\right)\left(F_{1} \Delta \otimes I_{\mathcal{F}}\right)\left(I_{\widehat{\mathrm{k}}} \otimes V_{s}^{(2)}\right)\left(\Delta F_{2} \otimes I_{\mathcal{F}}\right)-\left(I_{\widehat{\mathrm{k}}} \otimes V_{s}\right)\left(F_{1} \Delta F_{2} \otimes I_{\mathcal{F}}\right) .
\end{aligned}
$$


Therefore, by (2.5), $\mathbb{E}\left[V_{t}^{(1)} V_{t}^{(2)}-V_{t}\right]=\int_{0}^{t} \mathrm{~d} s \mathbb{E}\left[Y_{s}\right]$ where $Y=Y^{(1)}+Y^{(2)}+Y^{(3)}$ for the processes given by

$$
\begin{aligned}
Y_{s}^{(1)} & :=V_{s}^{(1)}\left(K_{1} \otimes I_{\mathcal{F}}\right) V_{s}^{(2)}-V_{s}\left(K_{1} \otimes I_{\mathcal{F}}\right) \\
Y_{s}^{(2)} & :=\left(V_{s}^{(1)} V_{s}^{(2)}-V_{s}\right)\left(K_{2} \otimes I_{\mathcal{F}}\right) \\
Y_{s}^{(3)} & :=V_{s}^{(1)}\left(M_{1} \otimes I_{\mathcal{F}}\right)\left(I_{\mathrm{k}} \otimes V_{s}^{(2)}\right)\left(L_{2} \otimes I_{\mathcal{F}}\right)-V_{s}\left(M_{1} L_{2} \otimes I_{\mathcal{F}}\right) .
\end{aligned}
$$

Note that, since $V^{(1)}, V^{(2)}$ and $V$ are contractive,

$$
\left\|\mathbb{E}\left[Y_{r}\right]\right\| \leqslant 2\left(\left\|K_{1}\right\|+\left\|K_{2}\right\|+\left\|M_{1}\right\|\left\|L_{2}\right\|\right)=2 C\left(F_{1}, F_{2}\right) \quad\left(r \in \mathbb{R}_{+}\right) .
$$

In turn, the quantum Itô formula, together with the identities (2.5) and (2.6), imply that, for $i=1,2,3$ and $s \in \mathbb{R}_{+}, \mathbb{E}\left[Y_{s}^{(i)}\right]=\int_{0}^{s} \mathrm{~d} r \mathbb{E}\left[Z_{r}^{(i)}\right]$ for processes given by

$$
\begin{aligned}
Z_{r}^{(1)}:= & V_{r}^{(1)}\left(\left(K_{1}\right)^{2} \otimes I_{\mathcal{F}}\right) V_{r}^{(2)}-V_{r}\left(\left(K_{1}\right)^{2} \otimes I_{\mathcal{F}}\right)+ \\
& V_{r}^{(1)}\left(K_{1} \otimes I_{\mathcal{F}}\right) V_{r}^{(2)}\left(K_{2} \otimes I_{\mathcal{F}}\right)-V_{r}\left(K_{1} K_{2} \otimes I_{\mathcal{F}}\right)+ \\
& V_{r}^{(1)}\left(M_{1} \otimes I_{\mathcal{F}}\right)\left(I_{\mathrm{k}} \otimes K_{1} \otimes I_{\mathcal{F}}\right)\left(I_{\mathrm{k}} \otimes V_{r}^{(2)}\right)\left(L_{2} \otimes I_{\mathcal{F}}\right)-V_{r}\left(M_{1} L_{2} K_{1} \otimes I_{\mathcal{F}}\right), \\
Z_{r}^{(2)}:= & Y_{r}\left(K_{2} \otimes I_{\mathcal{F}}\right), \text { and } \\
Z_{r}^{(3)}:= & V_{r}^{(1)}\left(K_{1} M_{1} \otimes I_{\mathcal{F}}\right)\left(I_{\mathrm{k}} \otimes V_{r}^{(2)}\right)\left(L_{2} \otimes I_{\mathcal{F}}\right) V_{r}^{(2)}-V_{r}\left(K_{2} M_{1} L_{2} \otimes I_{\mathcal{F}}\right)+ \\
& V_{r}^{(1)}\left(M_{1} \otimes I_{\mathcal{F}}\right)\left(I_{\mathrm{k}} \otimes V_{r}^{(2)}\right)\left(I_{\mathrm{k}} \otimes K_{2} \otimes I_{\mathcal{F}}\right)\left(L_{2} \otimes I_{\mathcal{F}}\right)-V_{r}\left(K_{2} M_{1} L_{2} \otimes I_{\mathcal{F}}\right)+ \\
& V_{r}^{(1)}\left(M_{1} \otimes I_{\mathcal{F}}\right)\left(I_{\mathrm{k}} \otimes M_{1} \otimes I_{\mathcal{F}}\right)\left(I_{\mathrm{k} \otimes \mathrm{k}} \otimes V_{r}^{(2)}\right)\left(I_{\mathrm{k}} \otimes L_{2} \otimes I_{\mathcal{F}}\right)\left(I_{\mathrm{k} \otimes \mathrm{k}} \otimes V_{r}^{(2)}\right)\left(L_{2} \otimes I_{\mathcal{F}}\right) \\
& -V_{r}\left(\left(M_{1} L_{2}\right)^{2} \otimes I_{\mathcal{F}}\right) .
\end{aligned}
$$

Now, by further use of the contractivity of the processes $V^{(1)}, V^{(2)}$ and $V$, together with the estimate (3.1),

$\left\|\mathbb{E}\left[Z_{r}^{(1)}\right]\right\| \leqslant 2\left(\left\|K_{1}\right\|^{2}+\left\|K_{1}\right\|\left\|K_{2}\right\|+\left\|M_{1}\right\|\left\|K_{1}\right\|\left\|L_{2}\right\|\right)=2\left\|K_{1}\right\| C\left(F_{1}, F_{2}\right)$, $\left\|\mathbb{E}\left[Z_{r}^{(2)}\right]\right\| \leqslant 2 C\left(F_{1}, F_{2}\right)\left\|K_{2}\right\|$, and $\left\|\mathbb{E}\left[Z_{r}^{(3)}\right]\right\| \leqslant 2\left(\left\|K_{1}\right\|\left\|M_{1}\right\|\left\|L_{2}\right\|+\left\|M_{1}\right\|\left\|K_{2}\right\|\left\|L_{2}\right\|+\left\|M_{1}\right\|^{2}\left\|L_{2}\right\|^{2}\right)=2\left\|M_{1}\right\|\left\|L_{2}\right\| C\left(F_{1}, F_{2}\right)$, and so

$$
\begin{aligned}
\left\|\mathbb{E}\left[V_{t}^{(1)} V_{t}^{(2)}-V_{t}\right]\right\| & =\left\|\int_{0}^{t} \mathrm{~d} s \int_{0}^{s} \mathrm{~d} r \mathbb{E}\left[Z_{r}^{(1)}+Z_{r}^{(2)}+Z_{r}^{(3)}\right]\right\| \\
& \leqslant \int_{0}^{t} \mathrm{~d} s \int_{0}^{s} \mathrm{~d} r 2 C\left(F_{1}, F_{2}\right)^{2}=t^{2} C\left(F_{1}, F_{2}\right)^{2},
\end{aligned}
$$

as required.

Lemma 3.2. Let $F_{1} \in \mathfrak{q c}_{\beta_{1}}(\widehat{\mathrm{k}} \otimes \mathfrak{h}), F_{2} \in \mathfrak{q} \mathfrak{c}_{\beta_{2}}(\widehat{\mathrm{k}} \otimes \mathfrak{h}), c^{\prime}, c \in \mathrm{k}$ and $r, t \in \mathbb{R}_{+}$with $t>r$. Then

$$
\left\|\Omega\left(c_{[r, t}^{\prime}, c_{[r, t}\right)\left(X_{r, t}^{F_{1}} X_{r, t}^{F_{2}}-X_{r, t}^{F_{1} \triangleleft F_{2}}\right)\right\| \leqslant(t-r)^{2} e^{(t-r)\left(\beta_{1}+\beta_{2}\right)} C\left(F_{c^{\prime}}^{*} \triangleleft F_{1}, F_{2} \triangleleft F_{c}\right)^{2} .
$$

Proof. By definition of the evolutions $\left(X_{r, t}^{G}\right)_{0 \leqslant r \leqslant t}$, for $G=F_{1}, F_{2}$ and $F_{1} \triangleleft F_{2}$, and identity 2.8a along with the remark following it,

$$
\text { LHS }=\left\|\Omega\left(c_{[0, t-r[}^{\prime}, c_{[0, t-r[}\right)\left(X_{t-r}^{F_{1}} X_{t-r}^{F_{2}}-X_{t-r}^{F_{1} \triangleleft F_{2}}\right)\right\|
$$

and so we may suppose without loss of generality that $r=0$. In this case, by the identity (2.2), Example 2.2 and the associativity of $\triangleleft$, the estimate follows immediately from Propositions 2.3 and 3.1 . 
Now let $\mathbb{S}$ denote the subspace of $L^{2}\left(\mathbb{R}_{+} ; \mathrm{k}\right)$ consisting of step functions, whose rightcontinuous versions we use when evaluating. For $F_{1}, F_{2} \in \mathfrak{b}(\widehat{\mathrm{k}} \otimes \mathfrak{h})$ and $g^{\prime}, g \in \mathbb{S}$, set

$$
C\left(g^{\prime}, F_{1}, F_{2}, g\right):=\max \left\{C\left(F_{c^{\prime}}^{*} \triangleleft F_{1}, F_{2} \triangleleft F_{c}\right): c^{\prime} \in \operatorname{Ran} g^{\prime}, c \in \operatorname{Ran} g\right\} .
$$

Lemma 3.3. Let $F_{1} \in \mathfrak{q c}_{\beta_{1}}(\widehat{\mathrm{k}} \otimes \mathfrak{h}), F_{2} \in \mathfrak{q c}_{\beta_{2}}(\widehat{\mathrm{k}} \otimes \mathfrak{h}), g^{\prime}, g \in \mathbb{S}$ and $r, t \in \mathbb{R}_{+}$with $t>r$. Then

$$
\left\|\Omega\left(g_{[r, t[}^{\prime}, g_{[r, t]}\right)\left(X_{r, t}^{F_{1}} X_{r, t}^{F_{2}}-X_{r, t}^{F_{1} \triangleleft F_{2}}\right)\right\| \leqslant(t-r)^{2} e^{(t-r)\left(\beta_{1}+\beta_{2}\right)} C\left(g_{[r, t[}^{\prime}, F_{1}, F_{2}, g_{[r, t]}\right)^{2} .
$$

Proof. Set $\beta:=\beta_{1}+\beta_{2}$ and let $\left\{r=t_{0}<t_{1}<\cdots<t_{n}<t_{n+1}=t\right\}$ be such that $g$ and $g^{\prime}$ are constant, say $c^{i}$ and $d^{i}$ respectively, on the interval $\left[t_{i}, t_{i+1}[\right.$, and, for $i=0, \cdots, n$, set $\Omega_{i}:=\Omega\left(g_{\left[t_{i}, t_{i+1}\right.}^{\prime}, g_{\left[t_{i}, t_{i+1}[\right.}\right)$

$$
X_{i}^{1}:=X_{t_{i}, t_{i+1}}^{F_{1}}, X_{i}^{2}:=X_{t_{i}, t_{i+1}}^{F_{2}} \text { and } X_{i}:=X_{t_{i}, t_{i+1}}^{F},
$$

noting that $\Omega_{i}:=\Omega\left(c_{\left[t_{i}, t_{i+1}[\right.}^{i}, d_{\left[t_{i}, t_{i+1}\right]}^{i}\right)$. Then, again using identity 2.8b and the subsequent remark, we see that

$$
\Omega\left(g_{[r, t[}^{\prime}, g_{[r, t]}\right)\left(X_{r, t}^{F_{1}} X_{r, t}^{F_{2}}-X_{r, t}^{F_{1} \triangleleft F_{2}}\right)=\overrightarrow{\prod_{0 \leqslant i \leqslant n}} \Omega_{i}\left(X_{i}^{1} X_{i}^{2}\right)-\prod_{0 \leqslant i \leqslant n} \Omega_{i}\left(X_{i}\right) .
$$

Therefore, using Lemma 3.2 ,

$$
\begin{aligned}
\text { LHS } & =\left\|\sum_{i=0}^{n}\left(\prod_{0 \leqslant p<i} \Omega_{p}\left(X_{p}\right) \Omega_{i}\left(X_{i}^{1} X_{i}^{2}-X_{i}\right) \overrightarrow{\prod_{i<p \leqslant n}} \Omega_{p}\left(X_{p}^{1} X_{p}^{2}\right)\right)\right\| \\
& \leqslant \sum_{i=0}^{n} e^{\beta\left(t_{i}-r\right)}\left\|\Omega_{i}\left(X_{i}^{1} X_{i}^{2}-X_{i}\right)\right\| e^{\beta\left(t-t_{i+1}\right)} \\
& \leqslant e^{\beta(t-r)} \sum_{i=1}^{n}\left(t_{i+1}-t_{i}\right)^{2} C\left(F_{c^{i}}^{*} \triangleleft F_{1}, F_{2} \triangleleft F_{d^{i}}\right)^{2} \leqslant \text { RHS } .
\end{aligned}
$$

By a partition $\mathcal{P}$ of $\mathbb{R}_{+}$we mean a sequence $\left(s_{n}\right)$ in $\mathbb{R}_{+}$which is strictly increasing and tends to infinity. Where convenient we identify a partition with its set of terms. For $S \subset \subset \mathbb{R}_{+}$, let $|S|$ denote the mesh of $\{0\} \cup S$, that is, $\max \left\{\left|t_{i}-t_{i-1}\right|: 1 \leqslant i \leqslant k\right\}$, where $S=\left\{t_{1}<\cdots<t_{n}\right\}$ and $t_{0}:=0$.

Lemma 3.4. Let $F_{1} \in \mathfrak{q c}_{\beta_{1}}(\widehat{\mathrm{k}} \otimes \mathfrak{h}), F_{2} \in \mathfrak{q c}_{\beta_{2}}(\widehat{\mathrm{k}} \otimes \mathfrak{h}), g^{\prime}, g \in \mathbb{S}$ and $r, t \in \mathbb{R}_{+}$with $t>r$. Let $\mathcal{P}$ be a partition of $\mathbb{R}_{+}$, let $\left.\left\{t_{1}<\cdots<t_{N-1}\right\}=\mathcal{P} \cap\right] r, t\left[\right.$ and set $t_{0}:=r$ and $t_{N}:=t$. Then

$$
\begin{aligned}
& \left\|\Omega\left(g_{[r, t[}^{\prime}, g_{[r, t]}\right)\left(\prod_{1 \leqslant j \leqslant N}^{\longrightarrow} X_{t_{j-1}, t_{j}}^{F_{1}} X_{t_{j-1}, t_{j}}^{F_{2}}-X_{r, t}^{F_{1} \triangleleft F_{2}}\right)\right\| \\
& \quad \leqslant|\mathcal{P} \cap[r, t]|(t-r) e^{(t-r)\left(\beta_{1}+\beta_{2}\right)} C\left(g_{[r, t[}^{\prime}, F_{1}, F_{2}, g_{[r, t]}\right)^{2} .
\end{aligned}
$$

Proof. Set $\beta:=\beta_{1}+\beta_{2}$ and, for $j=1, \cdots, N$, set $\Omega_{j}:=\Omega\left(g_{\left[t_{j-1}, t_{j}[\right.}^{\prime}, g_{\left[t_{j-1}, t_{j}\right.}[)\right.$,

$$
X_{j}^{1}:=X_{t_{j-1}, t_{j}}^{F_{1}}, X_{j}^{2}:=X_{t_{j-1}, t_{j}}^{F_{1}} \text { and } X_{j}:=X_{t_{j-1}, t_{j}}^{F_{1} \triangleleft F_{2}} .
$$


Then, using Lemma 3.3 (and arguing as in its proof), we see that

$$
\begin{aligned}
\mathrm{LHS} & =\left\|\sum_{j=1}^{N}\left(\prod_{1 \leqslant n<j} \Omega_{n}\left(X_{n}\right) \Omega_{j}\left(X_{j}^{1} X_{j}^{2}-X_{j}\right) \underset{j<n \leqslant N}{\longrightarrow} \Omega_{n}\left(X_{n}^{1} X_{n}^{2}\right)\right)\right\| \\
& \leqslant \sum_{j=1}^{N} e^{\beta\left(t_{j-1}-r\right)}\left\|\Omega_{j}\left(X_{j}^{1} X_{j}^{2}-X_{j}\right)\right\| e^{\beta\left(t-t_{j+1}\right)} \\
& \leqslant e^{\beta(t-r)} \sum_{j=1}^{N}\left(t_{j}-t_{j-1}\right)^{2} C\left(g_{\left[t_{j-1}, t_{j}[\right.}^{\prime}, F_{1}, F_{2}, g_{\left[t_{j-1}, t_{j}[\right.}\right)^{2} \\
& \leqslant e^{\beta(t-r)}|\mathcal{P} \cap[r, t]|(t-r) C\left(g_{[r, t[}^{\prime}, F_{1}, F_{2}, g_{[r, t]}\right)^{2},
\end{aligned}
$$

as required.

We may now prove the main result.

Theorem 3.5. Let $V^{1}$ and $V^{2}$ be quasicontractive elementary $Q S$ cocycles on $\mathfrak{h}$ with noise dimension space $\mathrm{k}$ and respective stochastic generators $F_{1}$ and $F_{2}$, and let $T \in \mathbb{R}_{+}$and $\varphi \in B(\mathcal{F})_{*}$. Then

$$
\sup _{0 \leqslant r \leqslant t \leqslant T}\left\|\left(\operatorname{id}_{B(\mathfrak{h})} \bar{\otimes} \varphi\right)\left(V_{r, t}^{\mathcal{P}, 1 \triangleleft 2}-X_{r, t}^{F_{1} \triangleleft F_{2}}\right)\right\| \rightarrow 0 \quad \text { as }|\mathcal{P} \cap[0, T]| \rightarrow 0 .
$$

The notation here is as follows. For a partition $\mathcal{P}$ of $\mathbb{R}_{+}$and $0 \leqslant r \leqslant t$,

$$
V_{r, t}^{\mathcal{P}, 1 \triangleleft 2}:=\prod_{1 \leqslant j \leqslant N}^{\longrightarrow} V_{t_{j-1}, t_{j}}^{1} V_{t_{j-1}, t_{j}}^{2}
$$

in which $\left.\left\{t_{1}<\cdots<t_{N-1}\right\}=\mathcal{P} \cap\right] r, t\left[, t_{0}:=r\right.$ and $t_{N}:=t$.

Proof. Set $\beta:=\beta_{0}\left(V^{1}\right)+\beta_{0}\left(V^{2}\right)$ and $V:=X^{F_{1} \triangleleft F_{2}}$. We may suppose without loss of generality that $\varphi$ is of the form $\omega_{\varpi\left(h^{\prime}\right), \varpi(h)}$ for some $h^{\prime}, h \in \mathbb{S}$, since such functionals are total in the Banach space $B(\mathcal{F})_{*}$ and $\left(V_{r, t}^{\mathcal{P}, 1 \triangleleft 2}-V_{r, t}\right)$ is uniformly bounded by $2 e^{T \max \{0, \beta\}}$ for $[r, t] \subset[0, T]$.

For any partition $\mathcal{P}$ of $\mathbb{R}_{+}$, Lemma 3.4 implies that there is a constant $C=C\left(\varphi, F_{1}, F_{2}\right)$ such that, for all subintervals $[r, t]$ of $[0, T]$,

$$
\left\|\left(\operatorname{id}_{B(\mathfrak{h})} \bar{\otimes} \varphi\right)\left(V_{r, t}^{\mathcal{P}, 1 \triangleleft 2}-V_{r, t}\right)\right\| \leqslant|\mathcal{P} \cap[0, T]| T e^{T \beta} C^{2} .
$$

The result follows.

Proposition 3.1 and Lemmas 3.2, 3.3 and 3.4 extend to the case of QS generators $F_{i}=\left[\begin{array}{ll}K_{i} & M_{i} \\ L_{i} & N_{i}\end{array}\right](i=1, \cdots, n)$, the constant $C\left(F_{1}, F_{2}\right)$ being replaced by

$$
\Delta^{\perp} f_{1} \triangleleft\left(f_{2} \triangleleft \cdots \triangleleft f_{n-1}\right) \triangleleft f_{n} \Delta^{\perp}
$$

where

$$
f_{i}=\left[\begin{array}{cc}
\left\|K_{i}\right\| & \left\|M_{i}\right\| \\
\left\|L_{i}\right\| & \left\|N_{i}\right\|
\end{array}\right] \in B(\widehat{\mathbb{C}}) \text { and } \Delta^{\perp}=\Delta_{\mathbb{C}}^{\perp}=\left[\begin{array}{ll}
1 & 0 \\
0 & 0
\end{array}\right] \in B(\widehat{\mathbb{C}}) .
$$

In this case the quantum Itô product formula allows $X_{t}^{F_{1}} \cdots X_{t}^{F_{n}}-I$ to be expressed as a sum of $2^{n}-1$ QS integrals, each of which may be paired with one of the $2^{n}-1$ terms arising from the expansion of $F_{1} \triangleleft \cdots \triangleleft F_{n}$ in the identity

$$
X_{t}^{F_{1} \triangleleft \cdots \triangleleft F_{n}}-I=\int_{0}^{t} X_{s}^{F_{1} \triangleleft \cdots \triangleleft F_{n}} \cdot\left(F_{1} \triangleleft \cdots \triangleleft F_{n}\right) \mathrm{d} \Lambda_{s} .
$$


As a consequence, Theorem 3.5 extends to quasicontractive elementary QS cocycles $V^{1}, \cdots, V^{n}$, with respective stochastic generators $F_{1}, \cdots, F_{n}$, as follows:

$$
\sup _{0 \leqslant r \leqslant t \leqslant T}\left\|\left(\operatorname{id}_{B(\mathfrak{h})} \bar{\otimes} \varphi\right)\left(V_{r, t}^{\mathcal{P}, 1 \triangleleft \cdots \triangleleft n}-X_{r, t}^{F_{1} \triangleleft \cdots \triangleleft F_{n}}\right)\right\| \rightarrow 0 \quad \text { as } \quad|\mathcal{P} \cap[0, T]| \rightarrow 0,
$$

in the notation

$$
V_{r, t}^{\mathcal{P}, 1 \triangleleft \cdots \triangleleft n}:=\prod_{1 \leqslant j \leqslant N}^{\longrightarrow} V_{t_{j-1}, t_{j}}^{1} \cdots V_{t_{j-1}, t_{j}}^{n} .
$$

Remarks. (i) Note that the QS cocycle $V=X^{F_{1} \triangleleft \cdots \triangleleft F_{n}}$ is contractive (respectively, isometric, or coisometric) provided that all of $V^{1}, \cdots, V^{n}$ are.

(ii) Whilst the convergence of Trotter products holds in the above hybrid norm-ultraweak topology, if $V$ is isometric and $V^{1}, \cdots, V^{n}$ are contractive (in particular, if $V^{1}, \cdots, V^{n}$ are isometric) then convergence also holds in the strong operator topology:

$$
\sup _{0 \leqslant r \leqslant t \leqslant T}\left\|\left(V_{r, t}^{\mathcal{P}, 1 \triangleleft \cdots \triangleleft n}-X_{r, t}^{F_{1} \triangleleft \cdots \triangleleft F_{n}}\right) \xi\right\| \rightarrow 0 \quad \text { as } \quad|\mathcal{P} \cap[0, T]| \rightarrow 0 \quad\left(T \in \mathbb{R}_{+}, \xi \in \mathfrak{h} \otimes \mathcal{F}\right) .
$$

This follows from the uniform continuity of the function

$$
[0, T]_{\leqslant}^{2} \rightarrow \mathfrak{h} \otimes \mathcal{F}, \quad(r, t) \mapsto V_{r, t} \xi \quad\left(\xi \in \mathfrak{h} \otimes \mathcal{F}, T \in \mathbb{R}_{+}\right) .
$$

We now revisit the case of QS cocycles with independent driving noise and show how to view it as a special case of the above theorem. Suppose therefore that the noise dimension space has orthogonal decomposition $\mathrm{k}=\mathrm{k}_{1} \oplus \cdots \oplus \mathrm{k}_{n}$. Denoting the symmetric Fock space over $L^{2}\left(\mathbb{R}_{+} ; \mathrm{k}_{i}\right)$ by $\mathcal{F}^{i}$, second quantisation of the natural isometry from $L^{2}\left(\mathbb{R}_{+} ; \mathrm{k}_{i}\right)$ to $L^{2}\left(\mathbb{R}_{+} ; \mathbf{k}\right)$ (see $(2.1)$ ), followed by ampliation, gives an isometry $\mathfrak{h} \otimes \mathcal{F}^{i} \rightarrow \mathfrak{h} \otimes \mathcal{F}$ which in turn induces a normal *-algebra monomorphism

$$
\Upsilon^{i}: B\left(\mathfrak{h} \otimes \mathcal{F}^{i}\right) \rightarrow B(\mathfrak{h} \otimes \mathcal{F}) \quad(i=1, \cdots, n) .
$$

Corollary 3.6 ( $\left[\mathrm{LS}_{1}\right)$. For $i=1, \cdots, n$, let $V^{i}$ be a quasicontractive elementary $Q S$ cocycle on $\mathfrak{h}$ with noise dimension space $\mathrm{k}_{i}$ and stochastic generator $F_{i}$. Then, for all $T \in \mathbb{R}_{+}$and $\varphi \in B(\mathcal{F})_{*}$,

$$
\sup _{0 \leqslant r \leqslant t \leqslant T}\left\|\left(\operatorname{id}_{B(\mathfrak{h})} \bar{\otimes} \varphi\right)\left(V_{r, t}^{\mathcal{P}, 1 \boxplus \cdots \boxplus n}-X_{r, t}^{F_{1} \boxplus \cdots \boxplus F_{n}}\right)\right\| \rightarrow 0 \text { as }|\mathcal{P} \cap[0, T]| \rightarrow 0 .
$$

The notation here is as follows. For a partition $\mathcal{P}$ of $\mathbb{R}_{+}$and $0 \leqslant r \leqslant t$, setting $\left\{t_{1}<\right.$ $\left.\left.\cdots<t_{N-1}\right\}=\mathcal{P} \cap\right] r, t\left[, t_{0}:=r\right.$ and $t_{N}:=t$,

$$
V_{r, t}^{\mathcal{P}, 1 \boxplus \cdots \boxplus n}:=\prod_{1 \leqslant j \leqslant N} \Upsilon^{1}\left(V_{t_{j-1}, t_{j}}^{1}\right) \cdots \Upsilon^{n}\left(V_{t_{j-1}, t_{j}}^{n}\right),
$$

Proof. Suppose first that $n=2$. Note that, in the notation (1.7), $\Upsilon^{1}\left(V_{s, s^{\prime}}^{1}\right)=X_{s, s^{\prime}}^{\iota\left(F_{1}\right)}$ and $\Upsilon^{2}\left(V_{s, s^{\prime}}^{2}\right)=X_{s, s^{\prime}}^{\iota^{\prime}\left(F_{2}\right)}\left(s, s^{\prime} \in \mathbb{R}_{+}, s<s^{\prime}\right)$. Therefore, in view of identity (1.8), for this case the corollary follows immediately from Theorem 3.5. The general case follows similarly via obvious extension of the notation (1.7) and corresponding identity (1.8).

\section{Maximal Gaussian component of a QS generator}

In this section it is shown that every QS generator $F \in \mathfrak{q} \mathfrak{c}(\widehat{\mathrm{k}} \otimes \mathfrak{h})$ enjoys a unique decomposition $F_{1} \boxplus F_{2}$ in which $F_{2}$ is 'pure Gaussian' and $F_{1}$ is 'wholly non-Gaussian', in senses to be defined below. This amounts to extracting a maximal Gaussian component of the generator and demonstrating its uniqueness. It chimes well with the way that Hunt's formula decomposes the generator of a Lévy process on a compact Lie group into a maximal Gaussian component and a jump part (albeit not exactly uniquely). 
To each contraction $C \in B(\mathrm{k} \otimes \mathfrak{h})$ we associate the following closed subspaces of k:

$$
\mathrm{k}_{\mathrm{g}}^{C}:=\left\{c \in \mathrm{k}:(C-I) E_{c}=0\right\}, \text { where } E_{c}:=|c\rangle \otimes I_{\mathfrak{h}},
$$

and

$$
\mathrm{k}_{\mathrm{p}}^{C}:=\overline{\operatorname{Lin}}\left\{\left(I_{\mathrm{k}} \otimes\langle u|\right) \xi: \xi \in \operatorname{Ran}(C-I)^{*}, u \in \mathfrak{h}\right\} .
$$

The former captures the subspace of all 'directions' in which $C$ acts as the identity operator; the latter is complementary (see below). The subscripts denote 'Gaussian' and 'preservation' parts.

Letting $J_{\mathrm{p}}^{C}$ denote the inclusion $\mathrm{k}_{\mathrm{p}}^{C} \rightarrow \mathrm{k}$, set

$$
C_{\mathrm{p}}:=\left(J_{\mathrm{p}}^{C} \otimes I_{\mathfrak{h}}\right)^{*} C\left(J_{\mathrm{p}}^{C} \otimes I_{\mathfrak{h}}\right),
$$

the compression of $C$ to $\mathrm{k}_{\mathrm{p}}^{C} \otimes \mathfrak{h}$.

Lemma 4.1. Let $C \in B(\mathbf{k} \otimes \mathfrak{h})$ be a contraction. Then the following hold:

(a) $\mathrm{k}=\mathrm{k}_{\mathrm{p}}^{C} \oplus \mathrm{k}_{\mathrm{g}}^{C}$.

(b) C has block matrix form $\left[\begin{array}{cc}C_{\mathrm{p}} & 0 \\ 0 & I_{\mathrm{g}}^{C}\end{array}\right]$, where $I_{\mathrm{g}}^{C}:=I_{\mathrm{k}_{\mathrm{g}}^{C} \otimes \mathfrak{h}}$.

(c) $\left(\mathrm{k}_{\mathrm{p}}^{C}\right)_{\mathrm{p}}^{C_{\mathrm{p}}}=\mathrm{k}_{\mathrm{p}}^{C}$.

Proof. Set $I_{\mathrm{p}}^{C}:=I_{\mathrm{k}_{\mathrm{p}}^{C} \otimes \mathfrak{h}}$.

(a) Let $c \in \mathrm{k}$. Then, for all $u \in \mathfrak{h}$,

$$
\left\langle c,\left(I_{\mathrm{k}} \otimes\langle u|\right)(C-I)^{*} \xi\right\rangle=0 \text { for all } \xi \in \mathrm{k} \otimes \mathfrak{h} \Longleftrightarrow(C-I)(c \otimes u)=0 .
$$

It follows that $c \in\left(\mathrm{k}_{\mathrm{p}}^{C}\right)^{\perp}$ if and only if $c \in \mathrm{k}_{\mathrm{g}}^{C}$, so (a) follows.

(b) Contraction operators $T$ on a Hilbert space $\mathrm{H}$ enjoy the following elementary property. In terms of the orthogonal decomposition $\mathrm{H}=\mathrm{K}^{\perp} \oplus \mathrm{K}$ where $\mathrm{K}:=\operatorname{Ker}(T-I), T$ has diagonal block matrix form $\left[\begin{array}{cc}* & 0 \\ 0 & I_{\mathfrak{K}}\end{array}\right]$. Since $\mathrm{k}_{\mathrm{g}}^{C} \otimes \mathfrak{h} \subset \operatorname{Ker}(C-I)$ it follows that, in terms of the orthogonal decomposition $\mathrm{k} \otimes \mathfrak{h}=\left(\mathrm{k}_{\mathrm{p}}^{C} \otimes \mathfrak{h}\right) \oplus\left(\mathrm{k}_{\mathrm{g}}^{C} \otimes \mathfrak{h}\right), C$ has the claimed block matrix form.

(c) By (a) it suffices to show that $\left(\mathrm{k}_{\mathrm{p}}^{C}\right)_{\mathrm{g}}^{C_{\mathrm{p}}}=\{0\}$. Accordingly let $e \in\left(\mathrm{k}_{\mathrm{p}}^{C}\right)_{\mathrm{g}}^{C_{\mathrm{p}}}$. Then $\left(C_{\mathrm{p}}-I_{\mathrm{p}}^{C}\right) E_{e}=0$ and so, by (b), $(C-I) E_{J_{\mathrm{p}}^{C} e}=0$, in other words $J_{\mathrm{p}}^{C} e \in \mathrm{k}_{\mathrm{g}}^{C}$. Thus $J_{\mathrm{p}}^{C} e \in \mathrm{k}_{\mathrm{p}}^{C} \cap \mathrm{k}_{\mathrm{g}}^{C}=\{0\}$, by (a). Since $J_{\mathrm{p}}^{C}$ is an inclusion this implies that $e=0$, as required.

Definition 4.2. Let $F \in \mathfrak{q} \mathfrak{c}(\widehat{\mathrm{k}} \otimes \mathfrak{h})$ with block matrix form $\left[{ }_{*}^{*} C_{-I}^{*}\right]$. We say that

(a) $F$ is Gaussian if $\mathrm{k}_{\mathrm{g}}^{C}=\mathrm{k}$, equivalently $C=I$ so that $F$ has block matrix form $\left[\begin{array}{cc}* * \\ * & 0\end{array}\right]$

(b) $F$ is wholly non-Gaussian if $\mathrm{k}_{\mathrm{p}}^{C}=\mathrm{k}$, equivalently $C_{\mathrm{p}}=C$;

(c) $F$ is pure Gaussian if it has block matrix form $\left[\begin{array}{cc}-\frac{1}{2} L^{*} L-L^{*} \\ L\end{array}\right]$, for some $L \in B(\mathfrak{h} ; \mathrm{k} \otimes$ $\mathfrak{h})$, equivalently $\left[\begin{array}{cc}-\frac{1}{2} M M^{*} & M \\ -M^{*} & 0\end{array}\right]$, for some $M \in B(\mathbf{k} \otimes \mathfrak{h} ; \mathfrak{h}) ;$

(d) $F$ is pure preservation if it has block matrix form $\left[\begin{array}{ll}0 & 0 \\ 0 & C-I\end{array}\right]$;

(e) $F$ is pure drift if it has block matrix form $\left[\begin{array}{ll}* & 0 \\ 0 & 0\end{array}\right]$, in other words $F \in \mathfrak{z}(\widehat{\mathrm{k}} \otimes \mathfrak{h})$. Write

$$
\mathfrak{g}(\widehat{\mathrm{k}} \otimes \mathfrak{h}), \mathfrak{w} \mathfrak{n}-\mathfrak{g}(\widehat{\mathrm{k}} \otimes \mathfrak{h}), \mathfrak{p} \mathfrak{g}(\widehat{\mathrm{k}} \otimes \mathfrak{h}) \text { and } \mathfrak{p} \mathfrak{p}(\widehat{\mathrm{k}} \otimes \mathfrak{h}),
$$

for the respective classes of Gaussian, wholly non-Gaussian, pure Gaussian and pure preservation generator.

Remarks. (i) The terminology has its origins in the work of Schürmann on Lévy-Khintchintype decompositions for Lévy processes on bialgebras such as algebraic quantum groups 
([Sch $]$. In the presence of a minimality condition, the Gaussian property for the generator of an elementary unitary QS cocycle $U$ may alternatively be expressed in terms of the cocycle itself as follows:

$$
t^{-1} \mathbb{E}\left[\left(U_{t}^{1}-I\right)_{v_{1}}^{u_{1}}\left(U_{t}^{2}-I\right)_{v_{2}}^{u_{2}}\left(U_{t}^{3}-I\right)_{v_{3}}^{u_{3}}\right] \rightarrow 0 \text { as } t \rightarrow 0
$$

for all $u_{1}, \cdots, v_{3} \in \mathfrak{h}$ and all choices of $U^{1}, U^{2}$ and $U^{3}$ from $\left\{U, U^{*}\right\}$ where, for $X \in$ $B(\mathfrak{h} \otimes \mathcal{F})$ and $u, v \in \mathfrak{h}, X_{v}^{u}:=\left(\omega_{u, v} \bar{\otimes} \operatorname{id}_{B(\mathcal{F})}\right)(X)=\left(\langle u| \otimes I_{\mathcal{F}}\right) X\left(|v\rangle \otimes I_{\mathcal{F}}\right)$ (see [SSS] and $[\mathrm{Sch}])$.

(ii) By Theorem $1.3(\mathrm{a}), F \in \mathfrak{g}(\widehat{\mathrm{k}} \otimes \mathfrak{h})$ if and only if it has block matrix form $\left[\begin{array}{cc}K & -L^{*} \\ L & 0\end{array}\right]$, for some $K \in B(\mathfrak{h})$ and $L \in B(\mathfrak{h} ; \mathrm{k} \otimes \mathfrak{h})$.

(ii) Given a Gaussian QS generator $\left[\begin{array}{cc}K & -L^{*} \\ L & 0\end{array}\right]$, any orthogonal decomposition $\mathrm{k}=\mathrm{k}_{1} \oplus \mathrm{k}_{2}$ determines a decomposition $F=F_{1} \boxplus F_{1}^{\prime}$ in which, corresponding to the block matrix decomposition $L=\left[\begin{array}{l}L_{1} \\ L_{2}\end{array}\right], F_{1}$ is pure Gaussian with

$$
F_{1}=\left[\begin{array}{cc}
-\frac{1}{2} L_{1}^{*} L_{1} & -L_{1}^{*} \\
L_{1} & 0
\end{array}\right] \text { and } F_{1}^{\prime}=\left[\begin{array}{cc}
K+\frac{1}{2} L_{1}^{*} L_{1} & -L_{2}^{*} \\
L_{2} & 0
\end{array}\right]
$$

Since $L^{*} L=L_{1}^{*} L_{1}+L_{2}^{*} L_{2}, F_{1}^{\prime}$ is pure Gaussian too if and only $F$ is.

(iii) In the case of no noise $(\mathrm{k}=\{0\}), \mathfrak{p} \mathfrak{p}(\widehat{\mathrm{k}} \otimes \mathfrak{h})=\emptyset$ and $\mathfrak{g}(\widehat{\mathrm{k}} \otimes \mathfrak{h}), \mathfrak{w} \mathfrak{n}-\mathfrak{g}(\widehat{\mathrm{k}} \otimes \mathfrak{h}), \mathfrak{p} \mathfrak{g}(\widehat{\mathrm{k}} \otimes \mathfrak{h})$ and $\mathfrak{z}(\widehat{k} \otimes \mathfrak{h})$ all equal $\mathfrak{b}(\widehat{k} \otimes \mathfrak{h})$. Otherwise, when $\mathfrak{k} \neq\{0\}$, all of $\mathfrak{g}(\widehat{\mathrm{k}} \otimes \mathfrak{h}), \mathfrak{w} \mathfrak{n}-\mathfrak{g}(\widehat{\mathrm{k}} \otimes \mathfrak{h})$, $\mathfrak{p} \mathfrak{g}(\widehat{\mathrm{k}} \otimes \mathfrak{h}), \mathfrak{p} \mathfrak{p}(\widehat{\mathrm{k}} \otimes \mathfrak{h})$ and $\mathfrak{z}(\widehat{\mathrm{k}} \otimes \mathfrak{h})$ are selfadjoint and the following relations are easily verified:

$$
\begin{aligned}
& \mathfrak{g}(\widehat{\mathrm{k}} \otimes \mathfrak{h}) \cap \mathfrak{w} \mathfrak{n}-\mathfrak{g}(\widehat{\mathrm{k}} \otimes \mathfrak{h})=\emptyset ; \\
& \mathfrak{p g}(\widehat{\mathrm{k}} \otimes \mathfrak{h}) \cap \mathfrak{z}(\widehat{\mathrm{k}} \otimes \mathfrak{h})=\{0\}=\mathfrak{g}(\widehat{\mathrm{k}} \otimes \mathfrak{h}) \cap \mathfrak{p} \mathfrak{p}(\widehat{\mathrm{k}} \otimes \mathfrak{h}) ; \\
& \mathfrak{p} \mathfrak{g}(\widehat{\mathrm{k}} \otimes \mathfrak{h}) \subset \mathfrak{u}(\widehat{\mathrm{k}} \otimes \mathfrak{h}) \text { and } \mathfrak{p} \mathfrak{p}(\widehat{\mathrm{k}} \otimes \mathfrak{h}) \subset \mathfrak{c}(\widehat{\mathrm{k}} \otimes \mathfrak{h}) ; \\
& \mathfrak{g}(\widehat{\mathrm{k}} \otimes \mathfrak{h}) \triangleleft \mathfrak{g}(\widehat{\mathrm{k}} \otimes \mathfrak{h})=\mathfrak{g}(\widehat{\mathrm{k}} \otimes \mathfrak{h}) \text { and } \mathfrak{p p}(\widehat{\mathrm{k}} \otimes \mathfrak{h}) \triangleleft \mathfrak{p p}(\widehat{\mathrm{k}} \otimes \mathfrak{h})=\mathfrak{p p}(\widehat{\mathrm{k}} \otimes \mathfrak{h}) ; \\
& \mathfrak{z}(\widehat{\mathrm{k}} \otimes \mathfrak{h}) \triangleleft \mathfrak{p} \mathfrak{g}(\widehat{\mathrm{k}} \otimes \mathfrak{h})=\mathfrak{g}(\widehat{\mathrm{k}} \otimes \mathfrak{h})=\mathfrak{p} \mathfrak{g}(\widehat{\mathrm{k}} \otimes \mathfrak{h}) \triangleleft \mathfrak{z}(\widehat{\mathrm{k}} \otimes \mathfrak{h}) .
\end{aligned}
$$

Also, in view of the series decompositions (Proposition 1.4),

$$
\begin{aligned}
& \mathfrak{z}(\widehat{\mathrm{k}} \otimes \mathfrak{h}) \triangleleft \mathfrak{p} \mathfrak{g}(\widehat{\mathrm{k}} \otimes \mathfrak{h}) \triangleleft \mathfrak{p} \mathfrak{p}(\widehat{\mathrm{k}} \otimes \mathfrak{h}) \supset \mathfrak{i}(\widehat{\mathrm{k}} \otimes \mathfrak{h}), \quad \text { and } \\
& \mathfrak{p} \mathfrak{p}(\widehat{\mathrm{k}} \otimes \mathfrak{h}) \triangleleft \mathfrak{p} \mathfrak{g}(\widehat{\mathrm{k}} \otimes \mathfrak{h}) \triangleleft \mathfrak{z}(\widehat{\mathrm{k}} \otimes \mathfrak{h}) \supset \mathfrak{i}(\widehat{\mathrm{k}} \otimes \mathfrak{h})^{*} .
\end{aligned}
$$

The next result implies that

$$
\mathfrak{q} \mathfrak{c}(\widehat{\mathrm{k}} \otimes \mathfrak{h})=\bigcup_{\mathrm{k}=\mathrm{k}_{1} \oplus \mathrm{k}_{2}} \mathfrak{w} \mathfrak{n}-\mathfrak{g}\left(\widehat{\mathrm{k}_{1}} \otimes \mathfrak{h}\right) \boxplus \mathfrak{p g}\left(\widehat{\mathrm{k}_{2}} \otimes \mathfrak{h}\right) .
$$

Theorem 4.3. Let $F \in \mathfrak{q} \mathfrak{c}(\widehat{\mathrm{k}} \otimes \mathfrak{h})$. Then $F$ enjoys a unique decomposition

$$
F_{\text {wn-g }} \boxplus F_{\mathrm{mg}}
$$

where, for some orthogonal decomposition $\mathrm{k}_{1} \oplus \mathrm{k}_{2}$ of $\mathrm{k}$,

$$
F_{\text {wn-g }} \in \mathfrak{w n}-\mathfrak{g}\left(\widehat{\mathrm{k}_{1}} \otimes \mathfrak{h}\right) \quad \text { and } \quad F_{\mathrm{mg}} \in \mathfrak{p g}\left(\widehat{\mathrm{k}_{2}} \otimes \mathfrak{h}\right) .
$$

Proof. Let $\left[\begin{array}{cc}K & M \\ L & C-I\end{array}\right]$ be the block matrix decomposition of $F$, and set $L_{\mathrm{g}}^{C}:=\left(J_{\mathrm{g}}^{C} \otimes I_{\mathfrak{h}}\right)^{*} L, L_{\mathrm{p}}^{C}:=\left(J_{\mathrm{p}}^{C} \otimes I_{\mathfrak{h}}\right)^{*} L, M_{\mathrm{g}}^{C}:=M\left(J_{\mathrm{g}}^{C} \otimes I_{\mathfrak{h}}\right)$ and $M_{\mathrm{p}}^{C}:=M\left(J_{\mathrm{p}}^{C} \otimes I_{\mathfrak{h}}\right)$.

Then $F=F_{\text {wn-g }} \boxplus F_{\text {mg }}$ where

$$
F_{\mathrm{wn}-\mathrm{g}}=\left[\begin{array}{cc}
K+\frac{1}{2}\left(L_{\mathrm{g}}^{C}\right)^{*} L_{\mathrm{g}}^{C} & M_{\mathrm{p}}^{C} \\
L_{\mathrm{p}}^{C} & C_{\mathrm{p}}-I
\end{array}\right] \text { and } F_{\mathrm{mg}}=\left[\begin{array}{cc}
-\frac{1}{2}\left(L_{\mathrm{g}}^{C}\right)^{*} L_{\mathrm{g}}^{C} & M_{\mathrm{g}}^{C} \\
L_{\mathrm{g}}^{C} & 0
\end{array}\right] .
$$


By Remark (ii) above, $M_{\mathrm{g}}^{C}=-\left(L_{\mathrm{g}}^{C}\right)^{*}$ so $F_{\mathrm{mg}}$ is pure Gaussian. It follows from Lemma 4.1 that $F_{\mathrm{wn}-\mathrm{g}}$ is wholly non-Gaussian. This proves existence.

For uniqueness, suppose that $F=F_{1} \boxplus F_{2}$ where $F_{1} \in \mathfrak{w n}-\mathfrak{g}\left(\widehat{\mathrm{k}_{1}} \otimes \mathfrak{h}\right)$ and $F_{2} \in \mathfrak{p g}\left(\widehat{\mathrm{k}_{2}} \otimes\right.$ h) for an orthogonal decomposition $\mathrm{k}=\mathrm{k}_{1} \oplus \mathrm{k}_{2}$. Then $F_{1}$ and $F_{2}$ have block matrix decompositions

moreover

$$
F_{1}=\left[\begin{array}{cc}
K_{1} & M_{1} \\
L_{1} & C_{1}-I
\end{array}\right] \text { and } F_{2}=\left[\begin{array}{cc}
-\frac{1}{2} L_{2}^{*} L_{2} & -L_{2}^{*} \\
L_{2} & 0
\end{array}\right]
$$

$$
\mathrm{k}_{\mathrm{p}}^{C_{1}}=\mathrm{k}_{1} \text { and } \mathrm{k}_{\mathrm{g}}^{C} \supset \mathrm{k}_{2} .
$$

Suppose that $e_{1} \in \mathrm{k}_{1}$ and $\left(\begin{array}{c}e_{1} \\ 0\end{array}\right) \in \mathrm{k}_{\mathrm{g}}^{C}$. Then $(C-I) E_{\left(\begin{array}{c}e_{1} \\ 0\end{array}\right)}=0$, so $\left(C_{1}-I\right) E_{e_{1}}=0$. Since $F_{1}$ is wholly non-Gaussian this implies that $e_{1}=0$. Thus $\mathrm{k}_{\mathrm{g}}^{C}=\mathrm{k}_{2}$ and $\mathrm{k}_{\mathrm{p}}^{C}=\mathrm{k}_{1}$. It follows that $C_{1}=C_{\mathrm{p}}$ and $L_{2}=L_{\mathrm{g}}^{C}$. This implies that $F_{1}=F_{\mathrm{wn}-\mathrm{g}}$ and $F_{2}=F_{\mathrm{mg}}$, as required.

Remarks. (i) Clearly $F_{\mathrm{mg}}$ is the maximal pure Gaussian component of $F$.

(ii) Let $F \in \mathfrak{i}(\widehat{\mathrm{k}} \otimes \mathfrak{h})$. Then the above decomposition of $F$ takes the form

$$
F=\left[\begin{array}{cc}
K_{1} & -L_{1}^{*} W \\
L_{1} & W-I
\end{array}\right] \boxplus\left[\begin{array}{cc}
-\frac{1}{2} L_{2}^{*} L_{2} & -L_{2}^{*} \\
L_{2} & 0
\end{array}\right] \in \mathfrak{i}\left(\widehat{\mathrm{k}_{1}} \otimes \mathfrak{h}\right) \boxplus \mathfrak{u}\left(\widehat{\mathrm{k}_{2}} \otimes \mathfrak{h}\right)
$$

with $\operatorname{Re} K_{1}=-\frac{1}{2} L_{1}^{*} L_{1}, W$ isometric and $\mathrm{k}_{\mathrm{p}}^{W}=\mathrm{k}_{1} ; W$ being unitary if and only if $F \in \mathfrak{u}(\widehat{\mathrm{k}} \otimes \mathfrak{h})$.

\section{Perturbation of QS COCycles}

In case the second of two elementary QS cocycles $V^{1}, V^{2}$ is isometric, there is another, more standard, way of realising the QS cocycle $X^{F_{1} \triangleleft F_{2}}$ where $F_{1}$ and $F_{2}$ are the stochastic generators of $V^{1}$ and $V^{2}$.

Theorem 5.1 (Cf. [EvH], [BLS]). Let $F_{1} \in \mathfrak{q} \mathfrak{c}(\widehat{\mathrm{k}} \otimes \mathfrak{h})$ and $F_{2} \in \mathfrak{i}(\widehat{\mathrm{k}} \otimes \mathfrak{h})$ Then

$$
X^{F_{1} \triangleleft F_{2}}=X^{j^{2}, F_{1}} X^{F_{2}}
$$

where $j^{2}$ is the normal ${ }^{*}$-monomorphic QS mapping cocycle on (the von Neumann algebra) $B(\mathfrak{h})$ given by

$$
j_{t}^{2}(x)=X_{t}^{F_{2}}\left(x \otimes I_{\mathcal{F}}\right)\left(X_{t}^{F_{2}}\right)^{*} \quad\left(x \in B(\mathfrak{h}), t \in \mathbb{R}_{+}\right),
$$

and $X^{j^{2}, F_{1}}$ is the unique strong solution of the $Q S$ differential equation $\mathrm{d} X_{t}=X_{t} \cdot G_{t} \mathrm{~d} \Lambda_{t}$, $X_{0}=I$, for the integrand process $G:=\left(\left(\mathrm{id}_{B(\widehat{\mathrm{k}})} \bar{\otimes} j_{t}^{2}\right)\left(F_{1}\right)\right)_{t \geqslant 0}$.

Proof. Given the existence of $X^{j^{2}, F_{1}}$ and its quasicontractivity ([BLS] $)$, the result follows easily from the quantum Itô product formula and uniqueness for weak solutions, which are bounded with locally uniform bounds, of the QS differential equation $\mathrm{d} X_{t}=X_{t}$. $\left(F_{1} \triangleleft F_{2}\right) \mathrm{d} \Lambda_{t}, X_{0}=I_{\mathfrak{h} \otimes \mathcal{F}}$ (see Theorem 2.1).

Remark. In [BLS] we worked in the equivalent category of QS right cocycles.

Applying this result to the Gaussian/non-Gaussian decomposition of QS generators (Theorem 4.3) yields the following result. Recall the injections (1.7) associated with realising the concatenation product in terms of the series product (1.8).

Corollary 5.2. Let $F \in \mathfrak{q} \mathfrak{c}(\widehat{\mathrm{k}} \otimes \mathfrak{h})$ with block matrix form $\left[\begin{array}{l}* \\ *{ }^{*}-I\end{array}\right]$. Then

$$
X^{F}=X^{j^{2}, F_{1}} X^{F_{2}}
$$

where, for the orthogonal decomposition $\mathrm{k} \otimes \mathfrak{h}=\left(\mathrm{k}_{\mathrm{p}}^{C} \otimes \mathfrak{h}\right) \oplus\left(\mathrm{k}_{\mathrm{g}}^{C} \otimes \mathfrak{h}\right)$,

$$
F_{1}=\iota\left(F_{\mathrm{wn}-\mathrm{g}}\right) \text { and } F_{2}=\iota^{\prime}\left(F_{\mathrm{mg}}\right) \text {. }
$$


Proof. The inclusions

$$
\iota^{\prime}\left(\mathfrak{p g}\left(\widehat{\mathrm{k}_{\mathrm{g}}^{C}} \otimes \mathfrak{h}\right)\right) \subset \mathfrak{p} \mathfrak{g}(\widehat{\mathrm{k}} \otimes \mathfrak{h}) \subset \mathfrak{u}(\widehat{\mathrm{k}} \otimes \mathfrak{h})
$$

ensure that $X^{F_{2}}$ is unitary and so Theorem 5.1 applies.

\section{Holomorphic QS Cocycles}

In this section the setting is extended to holomorphic QS cocycles $\left(\left[\mathrm{LS}_{2}\right]\right)$. Before formulating the conjecture, the corresponding Itô algebra is investigated, mirroring Section 1. As is customary, we identify each bounded Hilbert space operator $T$ with its associated quadratic form $q_{T}$, given by $q_{T}[\xi]:=\langle\xi, T \xi\rangle$. We also use the notation $q(\cdot, \cdot)$ for the sesquilinear form associated with a quadratic form $q[\cdot]$ by polarisation.

Fix Hilbert spaces $\mathrm{h}$ and $\mathrm{H}$. Let $\mathcal{Q}(\mathrm{h} \oplus \mathrm{H})$ denote the class of quadratic forms $\Gamma$ on $\mathrm{h} \oplus \mathrm{H}$ having the following structure:

$$
\left\{\begin{array}{l}
\operatorname{Dom} \Gamma=\mathcal{D} \oplus \mathrm{H} \\
\Gamma[\xi]=\gamma[u]-[\langle\zeta, L u\rangle+\langle\widetilde{L} u, \zeta\rangle+\langle\zeta,(C-I) \zeta\rangle], \quad \text { for } \xi=\left(\begin{array}{l}
u \\
\zeta
\end{array}\right) \in \operatorname{Dom} \Gamma,
\end{array}\right.
$$

where $\mathcal{D}$ is a subspace of $\mathrm{h}, C \in B(\mathrm{H})$, $\gamma$ is a quadratic form on $\mathrm{h}, L$ and $\widetilde{L}$ are operators from $\mathrm{h}$ to $\mathrm{H}$, and

$$
\operatorname{Dom} \gamma=\operatorname{Dom} L=\operatorname{Dom} \widetilde{L}=\mathcal{D} .
$$

For reasons which will become apparent, $\mathcal{D}$ is not assumed to be dense in $\mathrm{h}$.

Write $\Gamma \sim(\gamma, L, \widetilde{L}, C)$, and refer to $(\gamma, L, \widetilde{L}, C)$ as the components of $\Gamma$. Also define an associated operator on $\mathrm{h} \oplus \mathrm{H}$ by

$$
F_{\Gamma}^{\Delta}:=\left[\begin{array}{cc}
0 & 0 \\
L & C-I
\end{array}\right]
$$

Thus

$$
\operatorname{Dom} F_{\Gamma}^{\Delta}=\operatorname{Dom} \Gamma \text { and } \operatorname{Ran} F_{\Gamma}^{\Delta} \subset\{0\} \oplus \mathrm{H}=\operatorname{Ran} \Delta
$$

where, as usual, $\Delta:=0_{\mathrm{h}} \oplus I_{\mathrm{H}}$. The inclusion obviously implies that

$$
\operatorname{Ran} F_{\Gamma}^{\Delta} \subset \mathcal{D}^{\prime} \oplus \mathrm{H} \text { for any subspace } \mathcal{D}^{\prime} \text { of } \mathrm{h} \text {. }
$$

Note that if $\Gamma \in \mathcal{Q}(\mathbf{h} \oplus \mathrm{H})$ with components $(\gamma, L, \widetilde{L}, C)$, then the adjoint form $\Gamma^{*}$ belongs to $\mathcal{Q}(\mathrm{h} \oplus \mathrm{H})$ too, with

$$
\Gamma^{*} \sim\left(\gamma^{*}, \widetilde{L}, L, C^{*}\right) \text { and } F_{\Gamma^{*}}^{\Delta}=\left[\begin{array}{cc}
0 & 0 \\
\widetilde{L} & C^{*}-I
\end{array}\right] .
$$

Thus, in terms of the associated sesquilinear form,

$$
\left\langle\xi, F_{\Gamma}^{\Delta} \xi\right\rangle=-\Gamma(\Delta \xi, \xi) \text { and }\left\langle F_{\Gamma^{*}}^{\Delta} \xi, \xi\right\rangle=-\Gamma(\xi, \Delta \xi) \quad(\xi \in \operatorname{Dom} \Gamma) .
$$

Definition 6.1. For $\Gamma_{i} \in \mathcal{Q}(\mathbf{h} \oplus \mathrm{H})$, with components $\left(\gamma_{i}, L_{i}, \widetilde{L}_{i}, C_{i}\right)(i=1,2)$, define $\Gamma_{1} \triangleleft \Gamma_{2}, \Gamma_{1} \Delta \Gamma_{2} \in \mathcal{Q}(\mathrm{h} \oplus \mathrm{H})$ by

$\Gamma_{1} \triangleleft \Gamma_{2} \sim(\gamma, L, \widetilde{L}, C)$, where

$$
\begin{aligned}
& \gamma[u]=\gamma_{1}[u]+\gamma_{2}[u]-\left\langle\widetilde{L}_{1} u, L_{2} u\right\rangle, \\
& L=L_{1}+C_{1} L_{2}, \widetilde{L}=C_{2}^{*} \widetilde{L}_{1}+\widetilde{L}_{2} \text { and } C=C_{1} C_{2} ;
\end{aligned}
$$

$\Gamma_{1} \Delta \Gamma_{2} \sim(\gamma, L, \widetilde{L}, C)$, where

$$
\begin{aligned}
& \gamma[u]=-\left\langle\widetilde{L}_{1} u, L_{2} u\right\rangle, \\
& L=\left(C_{1}-I\right) L_{2}, \widetilde{L}=\left(C_{2}^{*}-I\right) \widetilde{L}_{1} \text { and } C=\left(C_{1}-I\right)\left(C_{2}-I\right)+I .
\end{aligned}
$$


Thus

$$
\begin{aligned}
& \Gamma_{1} \triangleleft \Gamma_{2}=\Gamma_{1}+\Gamma_{2}+\Gamma_{1} \Delta \Gamma_{2}, \\
& \operatorname{Dom}\left(\Gamma_{1} \triangleleft \Gamma_{2}\right)=\operatorname{Dom}\left(\Gamma_{1} \Delta \Gamma_{2}\right)=\operatorname{Dom} \Gamma_{1} \cap \operatorname{Dom} \Gamma_{2}, \\
& \left(\Gamma_{1} \Delta \Gamma_{2}\right)[\xi]=-\left\langle F_{\Gamma_{1}^{*}}^{\Delta} \xi, F_{\Gamma_{2}}^{\Delta} \xi\right\rangle \quad\left(\xi \in \operatorname{Dom} \Gamma_{1} \cap \operatorname{Dom} \Gamma_{2}\right), \text { and } \\
& F_{\Gamma_{1} \Delta \Gamma_{2}}^{\Delta}=\left(\{0\} \oplus\left(C_{1}-I\right)\right) F_{\Gamma_{2}}^{\Delta} .
\end{aligned}
$$

Lemma 6.2. The prescription $\left(\Gamma_{1}, \Gamma_{2}\right) \mapsto \Gamma_{1} \Delta \Gamma_{2}$ defines an associative product on the vector space $\mathcal{Q}(\mathrm{h} \oplus \mathrm{H})$ which is also bilinear and involutive:

$$
\left(\Gamma_{1} \Delta \Gamma_{2}\right)^{*}=\Gamma_{2}^{*} \Delta \Gamma_{1}^{*}
$$

Proof. Let $\Gamma_{i} \in \mathcal{Q}(\mathrm{h} \oplus \mathrm{H})$ with domain $\mathcal{D}_{i} \oplus \mathbf{H}(i=1,2,3)$. Bilinearity follows from the evident linearity of the map $\Gamma \mapsto F_{\Gamma}^{\Delta}$, and (6.4) holds since, for $\xi \in\left(\mathcal{D}_{1} \cap \mathcal{D}_{2}\right) \oplus \mathbf{H}$,

$$
\left(\Gamma_{1} \Delta \Gamma_{2}\right)^{*}[\xi]=\overline{\left(\Gamma_{1} \Delta \Gamma_{2}\right)[\xi]}=-\left\langle F_{\Gamma_{2}}^{\Delta} \xi, F_{\Gamma_{1}^{*}}^{\Delta} \xi\right\rangle=\left(\Gamma_{2}^{*} \Delta \Gamma_{1}^{*}\right)[\xi] .
$$

Clearly $\operatorname{Dom}\left(\left(\Gamma_{1} \Delta \Gamma_{2}\right) \Delta \Gamma_{3}\right)=\left(\mathcal{D}_{1} \cap \mathcal{D}_{2} \cap \mathcal{D}_{3}\right) \oplus \mathbf{H}=\operatorname{Dom}\left(\Gamma_{1} \Delta\left(\Gamma_{2} \Delta \Gamma_{3}\right)\right)$ and, for $\xi \in$ $\left(\mathcal{D}_{1} \cap \mathcal{D}_{2} \cap \mathcal{D}_{3}\right) \oplus \mathrm{H}$, 6.3c implies that

$$
\begin{aligned}
\left\langle F_{\Gamma_{1}^{*}}^{\Delta} \xi, F_{\Gamma_{2} \Delta \Gamma_{3}}^{\Delta} \xi\right\rangle & =\left\langle F_{\Gamma_{1}^{*}}^{\Delta} \xi,\left(\{0\} \oplus\left(C_{2}-I\right)\right) F_{\Gamma_{3}}^{\Delta} \xi\right\rangle \\
& =\left\langle\left(\{0\} \oplus\left(C_{2}^{*}-I\right)\right) F_{\Gamma_{1}^{*}}^{\Delta} \xi, F_{\Gamma_{3}}^{\Delta} \xi\right\rangle \\
& =\left\langle F_{\Gamma_{2}^{*} \Delta \Gamma_{1}^{*}}^{\Delta} \xi, F_{\Gamma_{3}}^{\Delta} \xi\right\rangle=\left\langle F_{\left(\Gamma_{1} \Delta \Gamma_{2}\right)^{*}}^{\Delta} \xi, F_{\Gamma_{3}}^{\Delta} \xi\right\rangle .
\end{aligned}
$$

Thus $\Delta$ is associative, by 6.3b.

Proposition 6.3. The composition $\left(\Gamma_{1}, \Gamma_{2}\right) \mapsto \Gamma_{1} \triangleleft \Gamma_{2}$ endows $\mathcal{Q}(\mathrm{h} \oplus \mathrm{H})$ with the structure of a ${ }^{*}$-monoid whose identity element is $\Gamma_{0} \sim(0,0,0, I)$, in particular,

$$
\left(\Gamma_{1} \triangleleft \Gamma_{2}\right)^{*}=\Gamma_{2}^{*} \triangleleft \Gamma_{1}^{*} .
$$

Proof. Let $\Gamma_{1}, \Gamma_{2}, \Gamma_{3} \in \mathcal{Q}(\mathrm{h} \oplus \mathrm{H})$. In view of Lemma 6.2.

$$
\left(\Gamma_{1}+\Gamma_{2}+\Gamma_{3}\right)+\left(\Gamma_{1} \Delta \Gamma_{2}+\Gamma_{2} \Delta \Gamma_{3}+\Gamma_{1} \Delta \Gamma_{3}\right)+\Gamma_{1} \Delta \Gamma_{2} \Delta \Gamma_{3}
$$

is a common expression for $\Gamma_{1} \triangleleft\left(\Gamma_{2} \triangleleft \Gamma_{3}\right)$ and $\left(\Gamma_{1} \triangleleft \Gamma_{2}\right) \triangleleft \Gamma_{3}$, which have common domain $\left(\mathcal{D}_{1} \cap \mathcal{D}_{2} \cap \mathcal{D}_{3}\right) \oplus \mathrm{H}$. It is easily seen that the element $\Gamma_{0} \sim(0,0,0, I)$ satisfies $\Gamma_{0} \triangleleft \Gamma=\Gamma=\Gamma \triangleleft \Gamma_{0}$ for all $\Gamma \in \mathcal{Q}(\mathbf{h} \oplus \mathrm{H})$. The fact that the adjoint operation defines an involution on the resulting monoid follows from its additivity on $\mathcal{Q}(\mathrm{h} \oplus \mathrm{H})$ and the identity (6.4).

For the following lemma, recall the range observation (6.1).

Lemma 6.4. Let $\Gamma_{i} \in \mathcal{Q}(\mathrm{h} \oplus \mathrm{H})$ with domain $\mathcal{D}_{i} \oplus \mathrm{H}(i=1,2,3)$. Then

$$
\left(\Gamma_{1} \triangleleft \Gamma_{2} \triangleleft \Gamma_{3}\right)[\xi]=\left(\Gamma_{1} \triangleleft \Gamma_{3}\right)[\xi]+\Gamma_{2}\left(\left(I+F_{\Gamma_{1}^{*}}^{\Delta}\right) \xi,\left(I+F_{\Gamma_{3}}^{\Delta}\right) \xi\right) \quad\left(\xi \in \mathcal{D}_{1} \cap \mathcal{D}_{2} \cap \mathcal{D}_{3}\right) .
$$

Proof. Let $\xi \in \mathcal{D}_{1} \cap \mathcal{D}_{2} \cap \mathcal{D}_{3}$. Then, since

$$
\Gamma_{1} \triangleleft \Gamma_{2} \triangleleft \Gamma_{3}-\Gamma_{1} \triangleleft \Gamma_{3}=\Gamma_{2}+\Gamma_{2} \Delta \Gamma_{3}+\Gamma_{1} \Delta \Gamma_{2}+\Gamma_{1} \Delta \Gamma_{2} \Delta \Gamma_{3},
$$

the lemma follows by several applications of the identities 6.2 and $6.3 \mathrm{c}$ :

$$
\begin{aligned}
\Gamma_{2} & \left(\left(I+F_{\Gamma_{1}^{*}}^{\Delta}\right) \xi,\left(I+F_{\Gamma_{3}}^{\Delta}\right) \xi\right) \\
& =\Gamma_{2}[\xi]+\Gamma_{2}\left(\xi, F_{\Gamma_{3}}^{\Delta} \xi\right)+\Gamma_{2}\left(F_{\Gamma_{1}^{*}}^{\Delta} \xi, \xi\right)+\Gamma_{2}\left(F_{\Gamma_{1}^{*}}^{\Delta} \xi, F_{\Gamma_{3}}^{\Delta} \xi\right) \\
& =\Gamma_{2}[\xi]-\left\langle F_{\Gamma_{2}^{*}}^{\Delta} \xi, F_{\Gamma_{3}}^{\Delta} \xi\right\rangle-\left\langle F_{\Gamma_{1}^{*}}^{\Delta} \xi, F_{\Gamma_{2}}^{\Delta} \xi\right\rangle-\left\langle F_{\Gamma_{1}^{*}}^{\Delta} \xi,\left(\{0\} \oplus\left(I-C_{2}\right)\right) F_{\Gamma_{3}}^{\Delta} \xi\right\rangle \\
& =\Gamma_{2}[\xi]+\left(\Gamma_{2} \Delta \Gamma_{3}\right)[\xi]+\left(\Gamma_{1} \Delta \Gamma_{2}\right)[\xi]+\left(\Gamma_{1} \Delta \Gamma_{2} \Delta \Gamma_{3}\right)[\xi] .
\end{aligned}
$$


Proposition 6.5. Let $\Gamma_{1}, \Gamma_{2} \in \mathcal{Q}(\mathrm{h} \oplus \mathrm{H})$ and $\beta_{1}, \beta_{2} \in \mathbb{R}$, and set $\Gamma=\Gamma_{1} \triangleleft \Gamma_{2}$ and $\beta=\beta_{1}+\beta_{2}$.

(a) Suppose that, for $i=1,2$,

$$
\Gamma_{i}^{*} \triangleleft \Gamma_{i} \geqslant 2 \beta_{i} \Delta^{\perp} \text { on } \operatorname{Dom} \Gamma_{i} .
$$

Then $\Gamma^{*} \triangleleft \Gamma \geqslant 2 \beta \Delta^{\perp}$ on $\operatorname{Dom} \Gamma$.

(b) Suppose that, for $i=1,2$,

$$
\Gamma_{i}^{*} \triangleleft \Gamma_{i}=0 \text { on } \operatorname{Dom} \Gamma_{i} .
$$

Then $\Gamma^{*} \triangleleft \Gamma=0$ on $\operatorname{Dom} \Gamma$.

Proof. By associativity and Lemma 6.4.

$$
\left(\Gamma^{*} \triangleleft \Gamma\right)[\xi]=\left(\Gamma_{2}^{*} \triangleleft \Gamma_{2}\right)[\xi]+\left(\Gamma_{1}^{*} \triangleleft \Gamma_{1}\right)\left[\left(I+F_{\Gamma_{2}}^{\Delta}\right) \xi\right]
$$

for all $\xi \in \operatorname{Dom} \Gamma_{1} \cap \operatorname{Dom} \Gamma_{2}$. The result therefore follows since $\Delta^{\perp} F_{\Gamma_{2}}^{\Delta}=0$ on Dom $\Gamma_{2}$.

Remark. Thus

$$
\left\{\Gamma \in \mathcal{Q}(\mathrm{h} \oplus \mathrm{H}): \Gamma^{*} \triangleleft \Gamma=0=\Gamma \triangleleft \Gamma^{*}\right\}
$$

forms a subgroup of the group of invertible elements of $(\mathcal{Q}(\mathrm{h} \oplus \mathrm{H}), \triangleleft)$.

To complete the discussion of the algebra of the series product on quadratic forms, here is the form generalisation of Proposition 1.2

Proposition 6.6. Let $\Gamma \in \mathcal{Q}(\mathrm{h} \oplus \mathrm{H})$. Then

$$
\left(\Gamma^{*} \triangleleft \Gamma\right)[\xi]=\left(\Gamma \triangleleft \Gamma^{*}\right)\left[\left(I+F_{\Gamma}^{\Delta}\right) \xi\right]+\left\|F_{\Gamma^{*} \triangleleft \Gamma}^{\Delta} \xi\right\|^{2} \quad(\xi \in \operatorname{Dom} \Gamma) .
$$

Let $\mathcal{V} \in \mathcal{Q}(\mathrm{h} \oplus \mathrm{H})$ be of the form $\nu \oplus 0_{\mathrm{H}}$ where $\nu \in \mathcal{Q}(\mathrm{h})_{\text {sa }}$. Then

$$
\Gamma^{*} \triangleleft \Gamma \geqslant \mathcal{V} \text { if and only if } \Gamma \triangleleft \Gamma^{*} \geqslant \mathcal{V} \text {. }
$$

Proof. Let $\xi=\left(\begin{array}{l}u \\ \zeta\end{array}\right) \in \operatorname{Dom} \Gamma$. Note that $\Gamma, \Gamma^{*}, \Gamma^{*} \triangleleft \Gamma, \Gamma \triangleleft \Gamma^{*}$ and $\Gamma^{*} \triangleleft \Gamma \triangleleft \Gamma^{*} \triangleleft \Gamma$ all share the same domain. On the one hand, setting $\Gamma_{1}=\Gamma_{2}=\Gamma^{*} \triangleleft \Gamma$ in 6.3a and 6.3b yields

$$
\left(\Gamma^{*} \triangleleft \Gamma \triangleleft \Gamma^{*} \triangleleft \Gamma\right)[\xi]-\left(\Gamma^{*} \triangleleft \Gamma\right)[\xi]=\left(\Gamma^{*} \triangleleft \Gamma\right)[\xi]-\left\|F_{\Gamma^{*} \triangleleft \Gamma}^{\Delta} \xi\right\|^{2} .
$$

On the other hand, setting $\Gamma_{1}=\Gamma^{*}, \Gamma_{2}=\Gamma \triangleleft \Gamma^{*}$ and $\Gamma_{3}=\Gamma$ in Lemma 6.4 yields

$$
\left(\Gamma^{*} \triangleleft \Gamma \triangleleft \Gamma^{*} \triangleleft \Gamma\right)[\xi]-\left(\Gamma^{*} \triangleleft \Gamma\right)[\xi]=\left(\Gamma \triangleleft \Gamma^{*}\right)\left[\left(I+F_{\Gamma}^{\Delta}\right) \xi\right] .
$$

Thus (6.5) holds.

Now suppose that $\Gamma \triangleleft \Gamma^{*} \geqslant \mathcal{V}$. Then, since

$$
\mathcal{V}\left[\left(I+F_{\Gamma}^{\Delta}\right) \xi\right]=\nu[u]=\mathcal{V}[\xi]
$$

(6.5) implies that $\left(\Gamma^{*} \triangleleft \Gamma\right)[\xi] \geqslant \mathcal{V}[\xi]$. Thus $\Gamma^{*} \triangleleft \Gamma \geqslant \mathcal{V}$. The converse implication follows by exchanging $\Gamma$ and $\Gamma^{*}$.

Now we return to the Hilbert spaces $\mathfrak{h}$ and k. Let $\mathfrak{X}_{\text {hol }}(\mathfrak{h})$ denote the class of quadratic forms $\gamma$ on $\mathfrak{h}$ which are closed, densely defined and satisfy the accretive and semisectorial conditions

$$
\operatorname{Re} \gamma+\beta \geqslant 0 \text { and }|\operatorname{Im} \gamma[u]| \leqslant \alpha\left(\operatorname{Re} \gamma[u]+\|u\|^{2}\right) \quad(u \in \operatorname{Dom} \gamma)
$$

for some $\beta \in \mathbb{R}$ and $\alpha \in \mathbb{R}_{+}$, and let $\mathcal{S}_{\text {hol }}(\mathfrak{h})$ denote the class of holomorphic semigroups they generate (see e.g. Ouh]).

In $\left[\mathrm{LS}_{2}\right.$, a quasicontractive QS cocycle $V$ on $\mathfrak{h}$ is called holomorphic if its expectation semigroup belongs to $\mathcal{S}_{\text {hol }}(\mathfrak{h})$. Denoting this class of QS cocycle by $\mathbb{Q S C}_{\text {hol }}(\mathfrak{h}, k)$, it is shown there that the correspondence $\mathfrak{X}_{\text {hol }}(\mathfrak{h}) \rightarrow \mathcal{S}_{\text {hol }}(\mathfrak{h})$ extends to a bijection

$$
\mathfrak{X}_{\text {hol }}^{4}(\mathfrak{h}, \mathfrak{k}) \rightarrow \mathbb{Q S C}_{\text {hol }}(\mathfrak{h}, \mathfrak{k}), \quad \Gamma \mapsto X^{\Gamma},
$$


in which $\mathfrak{X}_{\text {hol }}^{4}(\mathfrak{h}, \mathrm{k})$ denotes the subclass of $\mathcal{Q}(\widehat{k} \otimes \mathfrak{h})=\mathcal{Q}(\mathfrak{h} \oplus(\mathbf{k} \otimes \mathfrak{h}))$ consisting of forms $\Gamma \sim(\gamma, L, \widetilde{L}, C)$ such that $\gamma \in \mathfrak{X}_{\text {hol }}(\mathfrak{h})$ and $\Gamma^{*} \triangleleft \Gamma+2 \beta \Delta^{\perp} \geqslant 0$, for some $\beta \in \mathbb{R}$. We speak of the stochastic form generator of the holomorphic cocycle. If $\Gamma \sim(\gamma, L, \widetilde{L}, C) \in \mathfrak{X}_{\text {hol }}^{4}(\mathfrak{h}, \mathrm{k})$ then it follows from Proposition 6.6 that $\Gamma^{*} \in \mathfrak{X}_{\text {hol }}^{4}(\mathfrak{h}, \mathrm{k})$ and in $\left[\mathrm{LS}_{2}\right.$ it is also shown that $C \in B(\mathrm{k} \otimes \mathfrak{h})$ is a contraction, and $X^{\Gamma^{*}}=\left(X^{\Gamma}\right)^{\sharp}$, the dual QS cocycle defined in (2.7).

The bijection extends the above form-semigroup correspondence as follows: if $\Gamma \sim$ $(\gamma, 0,0, I)$ where $\gamma \in \mathfrak{X}_{\text {hol }}(\mathfrak{h})$ then $\Gamma \in \mathfrak{X}_{\text {hol }}^{4}(\mathfrak{h}, \mathbf{k})$ and $X^{\Gamma}=\left(P_{t} \otimes I_{\mathcal{F}}\right)_{t \geqslant 0}$ where $P$ is the holomorphic semigroup with form generator $\gamma$. It also extends that of Theorem 2.1, in the sense that if $F \in \mathfrak{q} \mathfrak{c}(\widehat{\mathrm{k}} \otimes \mathfrak{h})$ with block matrix form $\left[\begin{array}{cc}K & { }_{C} \\ C-I\end{array}\right]$ then $X^{F}=X^{\Gamma}$ for the form in $\mathfrak{X}_{\text {hol }}^{4}(\mathfrak{h}, \mathfrak{k})$ given by

$$
\Gamma \sim\left(-q_{K},-L,-M^{*}, C\right) .
$$

If $\Gamma_{1}, \Gamma_{2} \in \mathfrak{X}_{\text {hol }}^{4}(\mathfrak{h}, \mathfrak{k})$ then $\Gamma_{1} \triangleleft \Gamma_{2} \in \mathfrak{X}_{\text {hol }}^{4}(\mathfrak{h}, \mathfrak{k})$ provided only that Dom $\Gamma_{1} \cap \operatorname{Dom} \Gamma_{2}$ is dense. This neatly extends the fact that if $\gamma_{1}, \gamma_{2} \in \mathfrak{X}_{\text {hol }}(\mathfrak{h})$ then $\gamma_{1}+\gamma_{2} \in \mathfrak{X}_{\text {hol }}(\mathfrak{h})$ provided only that Dom $\gamma_{1} \cap \operatorname{Dom} \gamma_{2}$ is dense in $\mathfrak{h}$.

Conjecture 6.7. Let $V^{1}$ and $V^{2}$ be quasicontractive holomorphic $Q S$ cocycles on $\mathfrak{h}$ with noise dimension space $\mathrm{k}$ and respective stochastic form generators $\Gamma_{1}$ and $\Gamma_{2}$, and suppose that Dom $\Gamma_{1} \cap \operatorname{Dom} \Gamma_{2}$ is dense in $\widehat{\mathrm{k}} \otimes \mathfrak{h}$. Then (in the notation of Theorem 3.5), for all $T \in \mathbb{R}_{+}, \varphi \in B(\mathcal{F})_{*}$ and $u \in \mathfrak{h}$,

$$
\sup _{0 \leqslant r \leqslant t \leqslant T}\left\|\left(\operatorname{id}_{B(\mathfrak{h})} \bar{\otimes} \varphi\right)\left(V_{r, t}^{\mathcal{P}, 1 \triangleleft 2}-X_{r, t}^{\Gamma_{1} \triangleleft \Gamma_{2}}\right) u\right\| \rightarrow 0 \quad \text { as }|\mathcal{P} \cap[0, T]| \rightarrow 0 .
$$

Moreover, if the $Q S$ cocycle $X^{\Gamma_{1} \triangleleft \Gamma_{2}}$ is isometric and $V^{1}$ and $V^{2}$ are contractive then, for all $T \in \mathbb{R}_{+}$and $\xi \in \mathfrak{h} \otimes \mathcal{F}$,

$$
\sup _{0 \leqslant r \leqslant t \leqslant T}\left\|\left(V_{r, t}^{\mathcal{P}, 1 \triangleleft 2}-X_{r, t}^{\Gamma_{1} \triangleleft \Gamma_{2}}\right) \xi\right\| \rightarrow 0 \quad \text { as } \quad|\mathcal{P} \cap[0, T]| \rightarrow 0 .
$$

Remarks. The conjecture has three special cases where it is proven. Theorem 3.5 covers the case where $\Gamma_{1}$ and $\Gamma_{2}$ are bounded. In the semigroup case, where $\Gamma_{i} \sim\left(\gamma_{i}, 0,0, I\right)$ for $\gamma_{i} \in \mathfrak{X}_{\text {hol }}(\mathfrak{h})(i=1,2)$, it reduces to a version of a celebrated result of Kato - as extended by Simon ([Kat]). For the case of independent driving noises a version of the holomorphic counterpart to Corollary 3.6, which includes the Kato-Simon theorem, is proved in [LS $\mathrm{LS}_{3}$.

Acknowledgements. I am grateful to Mateusz Jurczyński and Michał Gnacik for useful comments on an earlier draft of the paper. Support from the UK-India Education and Research Initiative (UKIERI), under the research collaboration grant Quantum Probability, Noncommutative Geometry \& Quantum Information, is also gratefully acknowledged.

\section{REFERENCES}

[Bel] V.P. Belavkin, A new form and a *algebraic structure of quantum stochastic integrals in Fock space, Rend. Sem. Mat. Fis. Milano 58 (1988), 177-193.

[BGL] A.C.R. Belton, M. Gnacik and J.M. Lindsay, Strong convergence of quantum random walks via semigroup decomposition, Preprint.

[BLS] A.C.R. Belton, J.M. Lindsay and A.G. Skalski, Quantum Feynman-Kac perturbations, J. London Math. Soc. (2) 89 (2014) no. 1, 275-300.

[Bha] B.V.R. Bhat, Cocycles of CCR flows, Mem. Amer. Math. Soc. 149 (2001), no. 709.

$[\mathrm{BrR}]$ O. Bratteli and D.W. Robinson, "Operator Algebras and Quantum Statistical Mechanics II: Equilibrium states. Models in quantum statistical mechanics," 2nd Edition, Springer-Verlag, Berlin, 1997.

[Che] P. R. Chernoff, Product formulas, non-linear semigroups and addition of unbounded operators, Mem. Amer. Math. Soc. 140, 1974.

[DGS] B. Das, D. Goswami and K.B. Sinha, A homomorphism theorem and a Trotter product formula for quantum stochastic flows with unbounded coefficients, Comm. Math. Phys. 330 (2014) no. 2, $435-467$. 
[DLT] B.K. Das, J.M. Lindsay and O. Tripak, Sesquilinear quantum stochastic analysis in Banach space, J. Math. Anal. Applic. 409 (2014) no. 2, 1032-1051.

[Dav] E.B. Davies, "One-Parameter Semigroups," Academic Press, London, 1980.

[EvH] M.P. Evans and R.L. Hudson, Perturbations of quantum diffusions, J. London Math. Soc. (2) (1990), no. 2, 373-384.

[Fag] F. Fagnola, Characterization of isometric and unitary weakly differentiable cocycles in Fock space, in, "Quantum Probability \& Related Topics," QP-PQ VIII, (Ed. L. Accardi), World Scientific, Singapore, 1993, pp. 143-164.

[GL+] D. Goswami, J.M. Lindsay, K.B. Sinha and S.J. Wills, Dilation of Markovian cocycles on a von Neumann algebra, Pacific J. Math. 211 (2003) no. 2, 221-247.

[GoJ] J. Gough and M. James, The series product and its application to quantum feedforward and feedback networks, IEEE Trans. Automat. Control 54 (2009) no. 11, 2530-2544.

[HiP] E. Hille and R.S. Philips, "Functional Analysis and Semigroups," Coll. Publ. 31, American Mathematical Society, 1957.

[Hol] A.S. Holevo, Stochastic representation of quantum dynamical semigroups (Russian), [Translated in Proc. Steklov Math. Inst. (1992) no. 2, 145-154.] Trudy Mat. Inst. Steklov 191 (1989), 130-139.

[HuP] R.L. Hudson and K.R. Parthasarathy, Quantum Itô's formula and stochastic evolution, Comm. Math. Phys. 93 (1984) no. 3, 301-323.

[Jou] J.-L. Journé, Structure des cocycles markoviens sur l'espace de Fock, Probab. Theory Related Fields 75 (1987) no. 2, 291-316.

[Kat] T. Kato, Trotter's product formula for an arbitrary pair of self-adjoint contraction semigroups, in, "Topics in Functional Analysis (essays dedicated to M.G. Krein on the occasion of his 70th birthday)," Adv. in Math. Suppl. Stud. 3, Academic Press, London 1978, pp. 185-195.

[L] J.M. Lindsay, Quantum stochastic analysis — an introduction, in, "Quantum Independent Increment Process, I: From Classical Probability to Quantum Stochastic Calculus" (Eds. U. Franz \& M. Schürmann), Lecture Notes in Mathematics 1865, Springer-Verlag, Heidelberg 2005, pp. 181-271.

[LS $]$ J.M. Lindsay and K. B. Sinha, A quantum stochastic Lie-Trotter product formula, Indian J. Pure Appl. Math. 41 (2010) no. 1, 313-325.

$\left[\mathrm{LS}_{2}\right]$ - - Holomorphic quantum stochastic contraction cocycles, Preprint.

$\left[\mathrm{LS}_{3}\right]$ _ — , Trotter-Kato product formulae for quantum stochastic cocycles, in preparation.

$\left[\mathrm{LW}_{1}\right]$ J.M. Lindsay and S.J. Wills, Existence, positivity, and contractivity for quantum stochastic flows with infinite dimensional noise, Probab. Theory Rel. Fields 116 (2000) no. 4, 505-543.

$\left[\mathrm{LW}_{2}\right]$ — - Markovian cocycles on operator algebras, adapted to a Fock filtration, J. Funct. Anal. 178 (2000), no. 2, 269-305.

$\left[\mathrm{LW}_{3}\right]$ - - Quantum stochastic cocycles and completely bounded semigroups on operators spaces, Int. Math. Res. Notices (2014) no. 11, 3096-3139.

[Mey] P.-A. Meyer, "Quantum Probability for Probabilists" (2nd Edn.), Lecture Notes in Math. 1538, Springer-Verlag, Berlin 1995.

[Ouh] E.M. Ouhabaz, "Analysis of Heat Equations on Domains," London Mathematical Society Monographs 31, Princeton University Press, Princeton, 2005.

[Par] K.R. Parthasarathy, "An Introduction to Quantum Stochastic Calculus," Monographs in Mathematics 85, Birkhäuser, Basel 1992.

[PaS] K.R. Parthasarathy and K.B. Sinha, A random Trotter-Kato product formula, "Statistics and Probability: Essays in Honor of C.R. Rao," eds. G. Kallianpur, P.R. Krishnaiah E J.K. Ghosh North-Holland, Amsterdam, 1982, pp. 553-566.

[ReS] M. Reed and B. Simon, "Methods of Modern Mathematical Physics, I: Functional Analysis (2nd Edn.), II: Fourier Analysis, Self-Adjointness," Academic Press, New York, 1980, 1975.

[SSS] L. Sahu, M. Schürmann and K.B. Sinha, Unitary processes with independent increments and representations of Hilbert tensor algebras, Publ. Res. Inst. Math. Sci. 45 (2009) no. 3, 745-785.

[Sch] M. Schürmann, "White noise on bialgebras," Lecture Notes in Math. 1544, Springer-Verlag, Berlin 1993.

[Sko] A.V. Skorohod, "Asymptotic Methods in the Theory of Stochastic Differential Equations," Translations of Mathematical Monographs 78, American Mathematical Society, Providence, 1989.

[Tro] H.F. Trotter, On the product of semi-groups of operators, Proc. Amer. Math. Soc. 10 (1959), $545-551$.

[Wil] S.J. Wills, On the generators of quantum stochastic operator cocycles, Markov Process. Related Fields 13 (2007) no. 1, 191-211.

Department of Mathematics \& Statistics, Lancaster University, Lancaster LA1 4YF, UK

E-mail address: j.m.lindsay@lancs.ac.uk 